\title{
L-SPACES, LEFT-ORDERABILITY AND TWO-BRIDGE KNOTS
}

\author{
IDRISSA BA
}

January 10, 2018

\begin{abstract}
We show that the 3-fold cyclic branched cover of any genus 2 two-bridge knot $K_{[-2 q, 2 s,-2 t, 2 l]}$ is an L-space and its fundamental group is not left-orderable. Therefore the family of 3 -fold cyclic branched cover of any genus 2 two-bridge knot $K_{[-2 q, 2 s,-2 t, 2 l]}$ verifies the $L$-space conjecture. We also show that if $K_{[2 k,-2 l]}$ is a 2 -bridge knot with $k \geq 2, l>0$, then the fundamental group of the 5 -fold cyclic branched cover of $K_{[2 k,-2 l]}$ is not left-orderable, which will complete the proof that the fundamental group of the 5 -fold cyclic branched cover of any genus one two-bridge knot is not left-orderable.
\end{abstract}

\section{INTRODUCTION}

In this paper we study the L-space conjecture for the cyclic branched covers of low genus two-bridge knots.

A closed, connected 3-manifold $M$ is an $L$-space if it is a rational homolgy sphere with the property that $r k \widehat{H F}(M)=\operatorname{ord}\left(H_{1}(M, \mathbb{Z})\right)([\mathrm{OSz}],[\mathrm{OSz} 06])$.

A group $G$ is called left-orderable if there exists a strict total ordering $<$, of $G$ such that $g<h$ implies $f g<f h$ for all $f, g, h \in G$. By convention the trivial group is not left-orderable.

A closed, connected, orientable 3 -manifold $M$ is called a total $L$-space if it is an $L$-space whose fundamental group is not left-orderable.

Conjecture 1.1. (Conjecture 1 in $[\mathrm{BGW}]$ ) An irreducible rational homolgy 3 -sphere is an L-space if and only if its fundamental group is not left-orderable.

Let $K_{\left[a_{1}, a_{2}, \cdots, a_{m}\right]}$ denote the two-bridge knot of type $\frac{p}{q}$, where $\left[a_{1}, a_{2}, \cdots, a_{m}\right]$ is a continued fraction expansion for $\frac{p}{q}$. We follow the convention that $\frac{p}{q}=a_{1}+\frac{1}{a_{2}+\cdots+\frac{1}{a_{m}}}$. Every twobridge knot admits a continued fraction expansion with an even number of even parameters $\left[2 a_{1}, 2 b_{1}, 2 a_{2}, 2 b_{2}, \cdots, 2 a_{m}, 2 b_{m}\right]$. The two-bridge knots $K_{\left[2 a_{1}, 2 b_{1}, 2 a_{2}, 2 b_{2}, \cdots, 2 a_{m}, 2 b_{m}\right]}$ are of genus $m$, when the $a_{i}$ and $b_{i}$ are in $\mathbb{Z} \backslash\{0\}$. Every genus 2 two-bridge knot can be written as $K_{\left[2 a_{1}, 2 b_{1}, 2 a_{2}, 2 b_{2}\right]}$, where $a_{i}$ and $b_{i}$ are in $\mathbb{Z} \backslash\{0\}, i=1,2$.

If $K$ is a genus one, alternating knot, then $K$ is either a genus one two bridge knot or, up to mirroring, a pretzel knot $P(2 n+1,2 m+1,2 p+1)$ with $m, n, p$ positive integers ([BZ], Lemma 3.1 ). In the case where $K$ is a genus one two bridge knot, much work has been done to study the left-orderability of the fundamental groups of its cyclic branched covers $\pi_{1}\left(\Sigma_{n}(K)\right)$. In this 
direction, the fundamental group of the 2-fold branched cover of $K_{[2 k,-2 l]}$ is not left-orderable for $k>0$ and $l>0$, because $\Sigma_{2}\left(K_{[2 k,-2 l]}\right)$ is a lens space, and in [DPT] it is shown that the fundamental group of the 3 -fold cyclic branched cover of $K_{[2 k,-2 l]}$ is not left-orderable for $k>0$ and $l>0$. Gordon and Lidman [GL] showed that the fundamental group of the 4-fold cyclic branched cover of $K_{[2 k,-2 l]}$ is not left-orderable for $k>0$ and $l>0$. But this is false for $n$ sufficiently large by $[\mathrm{Hu}]$ and [Tra].

The knot $5_{1}$ corresponds to the two-bridge knot $K_{[-2,2,-2,2]}$. Since the 3 -fold cyclic branched cover of $5_{1}$ is the Poincaré homology sphere, it is a total $L$-space. Therefore we can ask the following question: Is the 3 -fold cyclic branched cover of the knots $K_{[-2 q, 2 s,-2 t, 2 l]}$ a total Lspace? In this paper we answer this question positively.

Theorem 1.2. The 3-fold cyclic branched cover of $K_{[-2 q, 2 s,-2 t, 2 l]}$ is a total L-space, where $q$, $s, t$ and $l \in \mathbb{Z} \backslash\{0\}$.

Theorem 1.3. For $k \geq 2, l>0$, the fundamental group of the 5-fold cyclic branched cover of $K_{[2 k,-2 l]}$ is not left-orderable.

Theorem 1.3 combines with Theorem 2 in [DPT] and the result of Mitsunori Hori see [Te], to imply the following corollary.

Corollary 1.4. The 5-fold cyclic branched cover of any genus one two-bridge knot is a total L-space.

The paper is organized as follows. In the second section we introduce some background material and notations. In section 3 we proof Theorem 1.3. In section 4 , we prove that the fundamental group of the 3-fold cyclic branched cover of any genus 2 two-bridge knot is not left-orderable. Finaly, in section 5 we prove that the 3 -fold cyclic branched cover of any genus 2 two-bridge knot is an L-space, thus completing the proof of Theorem 1.2.

Acknowledgment. I would like to thank my supervisor Professor Steven Boyer for drawing my attention to the topic of the current paper and his consistent encouragement and support.

\section{BACKGROUND NOTIONS, TERMINOLOGY, AND NOTATION}

In this section we define some basic notions which will be useful in this paper.

Let $K$ be an oriented knot in $\mathbb{S}^{3}$. Let $M_{K}$ be the exterior of $K$ and $S$ be a Seifert surface for $K$. Isotope $S$ so that $S \cap \partial M_{K}$ is a longitude of $K$ and let $F=S \cap M_{K}$. Let $C$ be a tubular neighborhood of $F$ in $M_{K}$. Then $C$ is homeomorphic to $F \times[-1,1]$. Let $Y:=M_{K}-\operatorname{int}(C)$. The boundary of $Y$ has two copies $F^{-} \cong F \times\{-1\}$ and $F^{+} \cong F \times\{1\}$. We have a triple $\left(Y, F^{+}, F^{-}\right)$. Consider $n$-copies of this, denoted by $\left(Y_{i}, F_{i}^{+}, F_{i}^{-}\right), i=0, \cdots, n-1$, and glue them together by identifying $F_{0}^{+} \subset Y_{0}$ with $F_{1}^{-} \subset Y_{1}, F_{1}^{+} \subset Y_{1}$ with $F_{2}^{-} \subset Y_{2}, \cdots, F_{n-2}^{+} \subset Y_{n-2}$ with $F_{n-1}^{-} \subset Y_{n-1}$ and $F_{n-1}^{+} \subset Y_{n-1}$ with $F_{0}^{-} \subset Y_{0}$. Call the resulting space $Y_{n}$. There is a regular covering map $g: Y_{n} \longrightarrow M_{K}$ and its group of deck transformations is isomorphic to $\mathbb{Z}_{n}$. The manifold $Y_{n}$ is called the $n$-fold cyclic cover of $M_{K}$ and its fundamental group isomorphic to 
$\operatorname{Ker}\left(\pi_{1}\left(M_{K}\right) \longrightarrow \mathbb{Z}_{n}\right)$. To construct the $n$-fold cyclic branched cover $\Sigma_{n}(K)$, we have to glue a solid torus $V \cong D^{2} \times \mathbb{S}^{1}$ to $Y_{n}$ by identifying the meridian $\partial D^{2} \times\{1\}$ of $V$ with the preimage of the meridian $\mu$ of $\partial M_{K}$ under $g: Y_{n} \longrightarrow M_{K}$. The manifold $\Sigma_{n}(K)$ is a closed oriented 3-manifold.

For the construction of the $n$-fold cyclic branched cover of an oriented link $L$ see [BBG].

Definition 2.1. Let $L$ be a link and $D$ a link diagram of $L$. Checkerboard color the regions of the complement of the diagram in $\mathbb{R}^{2}$. Assume that the unbounded region $X_{0}$ is colored white. The other white regions will be called by $X_{1}, X_{2}, \cdots, X_{n}$. To any crossing $p$ of L we associate the number $\chi(p)$ which is +1 or -1 according to the convention in the Figure 1.
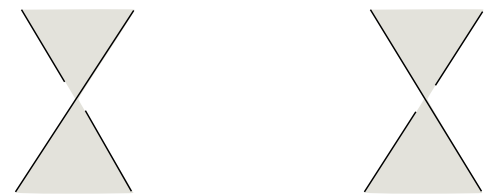

Figure 1. The signs convention +1 and -1 respectively.

Let $H=\left(h_{i j}\right)_{i, j=0,1, \cdots, n}$, where

$h_{i j}=\left\{\begin{array}{l}-\sum_{p} \chi(p), \text { if } i \neq j \text { and the summation extends over all crossings which connect } X_{i} \text { and } X_{j} \\ -\sum_{k=0 ; k \neq i}^{n} h_{i k} \text { if } i=j\end{array}\right.$

The matrix $H$ is called the unreduced Goeritz matrix of $D$. The Goeritz matrix $G$ of $D$ is obtained from $H$ by removing the first row and the first column of $H$.

Recall that the determinant of a link $L$ is the order of the first homology of its 2 -fold branched cover.

Theorem 2.2. Let $L$ be a non-split link. The determinant of $L$ is given by the absolute value of the determinant of $G(|\operatorname{det} G|)$.

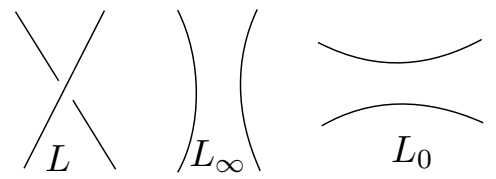

FiguRE 2. The resolutions $L_{\infty}$ and $L_{0}$ respectively.

Definition 2.3. The set $\mathcal{Q}$ of quasi-alternating links is the smallest set of links which satisfies the following properties:

(1) the unknot is in $\mathcal{Q}$,

(2) the set $\mathcal{Q}$ is closed under the following operation. Suppose $L$ is any link which admits a projection with a crossing with the following properties: 
- both resolution $L_{0}$ and $L_{\infty}$ are in $\mathcal{Q}$,

- $\operatorname{det}(L)=\operatorname{det}\left(L_{0}\right)+\operatorname{det}\left(L_{\infty}\right)$

then $L$ is in $\mathcal{Q}$.

Theorem 2.4. (Proposition 3.3 in [OSz]) The 2-fold branched cover of a quasi-alternating link $L$ is an L-space.

Let $L$ be a link, $L_{0}$ and $L_{\infty}$ be the links obtained by applying the resolutions as in Figure 2 such that $\operatorname{det}\left(L_{0}\right) \neq 0, \operatorname{det}\left(L_{\infty}\right) \neq 0$, and $\operatorname{det}(L)=\operatorname{det}\left(L_{0}\right)+\operatorname{det}\left(L_{\infty}\right)$. Then if $\Sigma_{2}\left(L_{0}\right)$ and $\Sigma_{2}\left(L_{\infty}\right)$ are $L$-spaces, then $\Sigma_{2}(L)$ is also an $L$-space ([OSz]).

\section{LEFT-ORDERABILITY AND GENUS ONE TWO-BRIDGE KNOTS}

In this section we will prove Theorem 1.3. The following Lemma will be important in the proof.

Lemma 3.1. Let $G$ be a left-orderable group which acts by order-preserving automorphisms on a totally ordered set $\left(X,<_{X}\right)$. Then for each $a \in X$, there exists a left-order $<$ on $G$ such that the stabilizer of $a, \operatorname{Stab}_{G}(a)$ is <-convex. The order $<$ is defined by:

$g \in P(<) \Leftrightarrow\left\{\begin{array}{l}g(a)>a, \text { or } \\ g(a)=a \text { and } 1<_{G} g\end{array}\right.$

Remark 3.2. A consequence of this definition is that if $g \geq h$, then $g(a) \geq h(a)$.

Proof of Lemma 3.1. Routine.

Let $G=\pi_{1}\left(\Sigma_{5}\left(K_{[2 k,-2 l]}\right)\right)$ for $k \geq 2, l>0$. We first note that $[2 k,-2 l]=2 k+\frac{1}{-2 l}=\frac{4 k l-1}{2 l}=$ $[2 k-1,1,2 l-1]$. By ([DPT], Proposition 2) we have that

$$
\pi_{1}\left(\Sigma_{n}\left(K_{[2 k+1,1,2 l+1]}\right)\right)=\left\{x_{1}, \cdots, x_{n} \mid r_{1}=1, \cdots, r_{n}=1, x_{1} x_{2} \cdots x_{n}=1\right\},
$$

where $k>0, l>0, r_{i}=x_{i}^{-1}\left(x_{i}^{-k} x_{i+1}^{k+1} x_{i}^{-1}\right)^{l} x_{i}^{-k} x_{i+1}^{k+1}\left(\left(x_{i+1}^{-k} x_{i+2}^{k+1} x_{i+1}^{-1}\right)^{l} x_{i+1}^{-k} x_{i+2}^{k+1}\right)^{-1}$ and $i \in \mathbb{Z} / n$. Replacing $k$ and $l$ by $k-1, l-1$ respectively, we get for each $i \in \mathbb{Z} / n$

$$
r_{i}=\left(x_{i}^{-k} x_{i+1}^{k}\right)^{l}\left(x_{i+2}^{-k} x_{i+1}^{k}\right)^{l-1}\left(x_{i+2}^{-k} x_{i+1}^{k-1}\right)
$$

Since there is an automorphism of $G$ given by sending $x_{i}$ to $x_{i+1}$, for each $i \in \mathbb{Z} / n$ and $K_{[2 k,-2 l]}$ is nontrivial no $x_{i}$ is trivial. Set $x=x_{1}, y=x_{2}, z=x_{3}, t=x_{4}, w=x_{5}$. We have

- $r_{0}=x y z t w=1$,

- $r_{1}=\left(x^{-k} y^{k}\right)^{l}\left(z^{-k} y^{k}\right)^{l-1}\left(z^{-k} y^{k-1}\right)=1$

- $r_{2}=\left(y^{-k} z^{k}\right)^{l}\left(t^{-k} z^{k}\right)^{l-1}\left(t^{-k} z^{k-1}\right)=1$

- $r_{3}=\left(z^{-k} t^{k}\right)^{l}\left(w^{-k} t^{k}\right)^{l-1}\left(w^{-k} t^{k-1}\right)=1$

- $r_{4}=\left(t^{-k} w^{k}\right)^{l}\left(x^{-k} w^{k}\right)^{l-1}\left(x^{-k} w^{k-1}\right)=1$

- $r_{5}=\left(w^{-k} x^{k}\right)^{l}\left(y^{-k} x^{k}\right)^{l-1}\left(y^{-k} x^{k-1}\right)=1$. 
We prove Theorem 1.3 by contradiction. Let $<$ be a left-order on $G$. Without loss of generality $x>1$. Here is the list of possible signs for $y, z, t, w$.

(1) $x>1, y>1, z>1, t>1, w>1$;

(2) $x>1, y<1, z>1, t>1, w>1$;

(3) $x>1, y<1, z<1, t>1, w>1$;

(4) $x>1, y<1, z<1, t<1, w>1$;

(5) $x>1, y<1, z<1, t<1, w<1$;

(6) $x>1, y>1, z<1, t>1, w>1$;

(7) $x>1, y>1, z<1, t<1, w>1$;

(8) $x>1, y>1, z<1, t<1, w<1$;

(9) $x>1, y>1, z>1, t<1, w>1$;

(10) $x>1, y>1, z>1, t<1, w<1$;

(11) $x>1, y>1, z>1, t>1, w<1$;

(12) $x>1, y<1, z>1, t>1, w<1$

(13) $x>1, y<1, z>1, t<1, w<1$;

(14) $x>1, y<1, z<1, t>1, w<1$;

(15) $x>1, y<1, z>1, t<1, w>1$;

(16) $x>1, y>1, z<1, t>1, w<1$.

We can immediately rule out eleven of these possibilities,

\begin{tabular}{|l|l|}
\hline Possibility & Ruled out by \\
\hline 1 & $r_{0}$ \\
\hline 2 & $r_{1}$ \\
\hline 5 & $r_{5}$ \\
\hline 6 & $r_{2}$ \\
\hline 9 & $r_{3}$ \\
\hline 11 & $r_{4}$ \\
\hline 12 & $r_{1}$ \\
\hline 13 & $r_{1}$ \\
\hline 14 & $r_{3}$ \\
\hline 15 & $r_{1}$ \\
\hline 16 & $r_{2}$ \\
\hline
\end{tabular}

TABLE 1

The cases which are left to check are (3), (4), (7), (8) and (10).

The automorphism $\phi$ of $G$ which sends $(x, y, z, t, w)$ to $(w, x, y, z, t)$ acts on $L O(G)$. Using $\phi$ the reader will verify that up to replacing an order by its opposite, an order of the form (3), (4), (7), (8) and (10) exists if and only if one of type $x>1, y>1, z<1, t<1, w<1$ exists. Assume that $<$ satisfies these inequalities. 
Lemma 3.3. $x^{-k} y^{k}<1, x^{k} y^{-k}<1$.

Proof. Since $z<1$ then $z^{-1}>1$. By relation $r_{1}$, since $y>1$, we have that $x^{-k} y^{k}<1$. By relation $r_{5}=\left(w^{-k} x^{k}\right)^{l}\left(y^{-k} x^{k}\right)^{l-1}\left(y^{-k} x^{k-1}\right)=1$ we have,

$$
\left(w^{-k} x^{k} w^{-k} x^{k} \ldots w^{-k} x^{k}\right)\left(y^{-k} x^{k} y^{-k} x^{k} \ldots y^{-k} x^{k}\right)\left(y^{-k} x^{k-1}\right)=1
$$

this implies

$$
w^{-k}\left(x^{k} w^{-k}\right)^{l-1}\left(x^{k} y^{-k}\right)^{l} x^{k-1}=1 .
$$

Since $w^{-1}>1$, and $x>1$ then $x^{k} y^{-k}<1$.

Lemma 3.4. $w x>1$ and $y z>1$.

Proof. Assume by contradiction that $w x<1$, then $x<w^{-1} \leq w^{-k}$, which implies that $x^{-1} w^{-k}>1$.

By $r_{5}=\left(w^{-k} x^{k}\right)^{l}\left(y^{-k} x^{k}\right)^{l-1}\left(y^{-k} x^{k-1}\right)=1$, we have,

$$
\left(w^{-k} x^{k}\right)\left(w^{-k} x^{k}\right)^{l-1}\left(y^{-k} x^{k}\right)^{l-1}\left(y^{-k} x^{k}\right) x^{-1}=1,
$$

which implies

$$
x^{k}\left(w^{-k} x^{k}\right)^{l-1}\left(y^{-k} x^{k}\right)^{l} x^{-1} w^{-k}=1 .
$$

Since $w^{-1}>1$, and $x>1$ then $y^{-k} x^{k}<1$, which implies $x^{-k} y^{k}>1$ this is a contradiction to Lemma 3.3.

Let show now that $y z>1$. By contradiction assume $y z<1$ then $z^{-1} y^{-1}>1$. By relation

$$
r_{1}=\left(x^{-k} y^{k}\right)^{l}\left(z^{-k} y^{k}\right)^{l-1}\left(z^{-k} y^{k-1}\right)=1
$$

we obtain

$$
\left(x^{-k} y^{k} x^{-k} y^{k} \ldots x^{-k} y^{k}\right)\left(z^{-k} y^{k}\right)^{l-1}\left(z^{-k} y^{k-1}\right)=1
$$

which implies

$$
\left(y^{k} x^{-k}\right)^{l-1} y^{k}\left(z^{-k} y^{k}\right)^{l-1} z^{-k} y^{k-1} x^{-k}=1
$$

By Lemma 3.3 we obtain $x^{k} y^{-k}<1$, which implies $y^{-k}<x^{-k}$, by multiplying in both side by $\left(y^{k} x^{-k}\right)^{l-1} y^{k}\left(z^{-k} y^{k}\right)^{l-1} z^{-k} y^{k-1}$ we have that,

$$
\left(y^{k} x^{-k}\right)^{l-1} y^{k}\left(z^{-k} y^{k}\right)^{l-1} z^{-k} y^{k-1} y^{-k}<1
$$

which implies

$$
\left(y^{k} x^{-k}\right)^{l-1} y^{k}\left(z^{-k} y^{k}\right)^{l-1} z^{-(k-1)} z^{-1} y^{-1}<1
$$

which is a contradiction since the terms on the left-hand side are positive. Thus $y z>1$.

Lemma 3.5. $z^{k} t^{-k}>1, t^{-k} z^{k}>1$, and $t^{k-1}>w^{k}>t^{k}$. 
Proof. By $r_{2}=\left(y^{-k} z^{k}\right)^{l}\left(t^{-k} z^{k}\right)^{l-1}\left(t^{-k} z^{k-1}\right)=1$ we have

$$
\left(y^{-k} z^{k} y^{-k} z^{k} \ldots y^{-k} z^{k}\right)\left(t^{-k} z^{k}\right)^{l-1}\left(t^{-k} z^{k-1}\right)=1
$$

which implies

$$
y^{-k}\left(z^{k} y^{-k}\right)^{l-1}\left(z^{k} t^{-k}\right)^{l} z^{k-1}=1 .
$$

Since $y^{-1}<1$ and $z<1$, then $z^{k} t^{-k}>1$.

Again by $r_{2}=\left(y^{-k} z^{k}\right)^{l}\left(t^{-k} z^{k}\right)^{l-1}\left(t^{-k} z^{k-1}\right)=1$ we obtain,

$$
y^{-k} z^{k}\left(y^{-k} z^{k}\right)^{l-1}\left(t^{-k} z^{k}\right)^{l} z^{-1}=1
$$

which implies

$$
z^{k}\left(y^{-k} z^{k}\right)^{l-1}\left(t^{-k} z^{k}\right)^{l} z^{-1} y^{-k}=1 .
$$

Since $y z>1$ by Lemma 3.4, then $z>y^{-1} \geq y^{-k}$, which implies $1>z^{-1} y^{-k}$. Therefore, $t^{-k} z^{k}>1$.

Now it left to show that $t^{k-1}>w^{k}>t^{k}$. By

$$
r_{4}=\left(t^{-k} w^{k}\right)^{l}\left(x^{-k} w^{k}\right)^{l-1}\left(x^{-k} w^{k-1}\right)=1
$$

since $x^{-1}<1$ and $w<1$, then $t^{-k} w^{k}>1$, which implies that $t^{k}<w^{k}$ and $w^{-k} t^{k}<1$. Since $t^{-k} z^{k}>1$ then $z^{-k} t^{k}<1$, and by relation

$$
r_{3}=\left(z^{-k} t^{k}\right)^{l}\left(w^{-k} t^{k}\right)^{l-1}\left(w^{-k} t^{k-1}\right)=1
$$

we obtain $w^{-k} t^{k-1}>1$. Thus, $t^{k-1}>w^{k}$.

Lemma 3.6. $t^{-1}>w^{-k+1} x^{k}$ and $w^{-k}<t^{-k}$.

Proof. The equation

$$
r_{3}=\left(z^{-k} t^{k}\right)^{l}\left(w^{-k} t^{k}\right)^{l-1}\left(w^{-k} t^{k-1}\right)=1
$$

implies

$$
A=t^{-1}\left(z^{-k} t^{k}\right)^{l}\left(w^{-k} t^{k}\right)^{l}=1
$$

multiplying $A$ with

$$
r_{4}=\left(t^{-k} w^{k}\right)^{l}\left(x^{-k} w^{k}\right)^{l-1}\left(x^{-k} w^{k-1}\right)=1
$$

we obtain

$$
A r_{4}=t^{-1}\left(z^{-k} t^{k}\right)^{l}\left(x^{-k} w^{k}\right)^{l-1}\left(x^{-k} w^{k-1}\right)=1
$$

which implies

$$
\left(z^{-k} t^{k}\right)^{l}\left(x^{-k} w^{k}\right)^{l-1}\left(x^{-k} w^{k-1} t^{-1}\right)=1 .
$$

Since $z^{-k} t^{k}<1$ by Lemma 3.5, and $x^{-k} w^{k}<1$ because $x^{-1}<1$ and $w<1$, then $x^{-k} w^{k-1} t^{-1}>$ 1. Therefore $t^{-1}>w^{-k+1} x^{k}$.

Now, let show that $w^{-k}<t^{-k}$. The equation

$$
r_{5}=\left(w^{-k} x^{k}\right)^{l}\left(y^{-k} x^{k}\right)^{l-1}\left(y^{-k} x^{k-1}\right)=1
$$

implies

$$
w^{-k} x^{k}\left(w^{-k} x^{k}\right)^{l-1}\left(y^{-k} x^{k}\right)^{l} x^{-1}=1
$$


which implies

$$
x^{-1} w^{-k} x^{k}\left(w^{-k} x^{k}\right)^{l-1}\left(y^{-k} x^{k}\right)^{l}=1
$$

Now, $w^{-k} x^{k}>1$, and by Lemma $3.3 y^{-k} x^{k}>1$. Therefore $x^{-1} w^{-k} x^{k}<1$. This implies $1<x^{k}<w^{k} x$. Hence $x>w^{-k}, x^{k}>x>w^{-k}>w^{-1}$ and $x^{k}>w^{-1}$. Multiplying both sides of $x^{k}>w^{-1}$ by $w^{-k+1}$ we have $w^{-k+1} x^{k}>w^{-k+1} w^{-1}=w^{-k}$, and since $t^{-1}>w^{-k+1} x^{k}$ then $t^{-k}>t^{-1}>w^{-k+1} x^{k}>w^{-k}$. Therefore $w^{-k}<t^{-k}$.

Remark 3.7. Lemmas 3.3, 3.4, 3.5, 3.6 hold for all lo's for which $1<x, 1<y, z<1, t<$ $1, w<1$. In particular, they will apply to $<_{c}$ in the Proof of Teorem 1.3.

Since $G$ is a countable left-orderable group, it can be seen as a subgroup of $\mathrm{Homeo}_{+}(\mathbb{R})$ and it will act effectively on $\mathbb{R}$ by order preserving homeomorphisms without global fixed points.

Lemma 3.8. Let $a$ be a fixed point for $x$. Then the only possible signs for $x, y, z, t, w$ with respect to $<_{a}$ are $t<_{a} 1, w<_{a} 1,1<_{a} x, 1<_{a} y, 1<_{a} z$.

Proof. Since $x>1$ and $x(a)=a$, we have $1<_{a} x$. Therefore as in the analysis of (1) through (16) above, one can see that the cases which are left to check are

(1) $y<_{a} 1, z<_{a} 1,1<_{a} t, 1<_{a} w, 1<_{a} x$

(2) $1<_{a} w, 1<_{a} x, y<_{a} 1, z<_{a} 1, t<_{a} 1$

(3) $z<_{a} 1, t<_{a} 1,1<_{a} w, 1<_{a} x, 1<_{a} y$

(4) $1<_{a} x, 1<_{a} y, z<_{a} 1, t<_{a} 1, w<_{a} 1$

(5) $t<_{a} 1, w<_{a} 1,1<_{a} x, 1<_{a} y, 1<_{a} z$

For (1), let $<^{\prime}=<_{a}^{o p}$, using Lemma 3.5 and replacing $x$ by $y, y$ by $z, z$ by $t, t$ by $w$ and $w$ by $x$, we have $w^{k}<^{\prime} x^{k}<^{\prime} w^{k-1}$. Therefore $w^{k-1}<_{a} x^{k}<_{a} w^{k}$, which implies $w(a)=a$, which is impossible because $w^{-1}(a)=a$ and $w^{-1}>1$ implies that $1<_{a} w^{-1}$, which is a contradiction to the fact that $1<_{a} w$. Therefore case (1) is not possible.

For (2), using Lemma 3.3 and replacing $x$ by $w, y$ by $x, z$ by $y, t$ by $z$ and $w$ by $t$, we have $x^{k}<_{a} w^{k}, x^{-k}<_{a} w^{-k}$. Hence $w(a)=a$ and by the same argument as for (1) we get a contradiction. Therefore case (2) is not possible.

For (3), let $<^{\prime}=<_{a}^{o p}$, using Lemma 3.5 and replacing $x$ by $z, y$ by $t, z$ by $w, t$ by $x$ and $w$ by $y$, we have $x^{k}<^{\prime} w^{k}$, hence $w^{k}<_{a} x^{k}$ and $w^{k}(a) \leq a$, and since $1<_{a} w$ then $w(a)=a$ and by the same argument as for (1) we get a contradiction. Therefore case (3) is not possible.

For (4), Lemma 3.3 implies $x^{-k} y^{k}(a) \leq a, x^{k} y^{-k}(a) \leq a$, hence $a=x^{-k}(a) \geq y^{-k}(a)$ and $y^{k}(a) \leq x^{k}(a)=a$. This two inequalities imply that $y(a)=a$. Therefore, by relation

$$
r_{5}=\left(w^{-k} x^{k}\right)^{l}\left(y^{-k} x^{k}\right)^{l-1}\left(y^{-k} x^{k-1}\right)=1
$$

we obtain that $\left(w^{-k} x^{k}\right)^{l}(a)=a$, which implies $\left(w^{-k} x^{k}\right)(a)=a$. Hence $w^{-k}(a)=a$, and $w(a)=a$. A similar argument using relation $r_{4}$ shows that $t(a)=a$. Since $x y z t w=1$, 
$z(a)=a$. Thus $a$ is fixed by $G$, which contradicts our assumptions. Therefore case (4) is not possible.

Lemma 3.9. Let $b$ be a fixed point for $y$. Then the only possible signs for $x, y, z, t, w$ with respect to $<_{b}$ are $w<_{b} 1, x<_{b} 1,1<_{b} y, 1<_{b} z, 1<_{b} t$.

Proof. Since $y>1$ and $y(b)=b$, we have $1<_{b} y$. Therefore as in the analysis of (1) through (16) above, by replacing $x$ by $y, y$ by $z, z$ by $t, t$ by $w, w$ by $x$ and $<$ by $<_{b}$, one can see that the cases which are left to check are

(1) $t<_{b} 1, w<_{b} 1,1<_{b} x, 1<_{b} y, 1<_{b} z$

(2) $1<_{b} y, 1<_{b} z, t<_{b} 1, w<_{b} 1, x<_{b} 1$

(3) $z<_{b} 1, t<_{b} 1,1<_{b} w, 1<_{b} x, 1<_{b} y$

(4) $1<_{b} x, 1<_{b} y, z<_{b} 1, t<_{b} 1, w<_{b} 1$

(5) $w<_{b} 1, x<_{b} 1,1<_{b} y, 1<_{b} z, 1<_{b} t$.

For (1), let $<^{\prime}=<_{b}^{o p}$, using Lemma 3.5 and replacing $x$ by $t, y$ by $w, z$ by $x, t$ by $y$ and $w$ by $z$, we have $y^{k}<^{\prime} z^{k}<^{\prime} y^{k-1}$. Therefore $y^{k-1}<_{b} z^{k}<_{b} w^{k}$, which implies $z(b)=b$, which is impossible because $z^{-1}(b)=b$ and $z^{-1}>1$ implies that $1<_{b} z^{-1}$, which is a contradiction to the fact that $1<_{b} z$. Therefore case (1) is not possible.

For (2), using Lemma 3.3 and replacing $x$ by $y, y$ by $z, z$ by $t, t$ by $w$ and $w$ by $x$, we have $z^{k}<_{b} y^{k}, z^{-k}<_{b} y^{-k}$. Hence $z(b)=b$ and by the same argument as for (1) we get a contradiction. Therefore case (2) is not possible.

For (3), let $<^{\prime}=<_{b}^{o p}$, using Lemma 3.5 and replacing $x$ by $z, y$ by $t, z$ by $w, t$ by $x$ and $w$ by $y$, we have $x^{k}<^{\prime} y^{k}<^{\prime} x^{k-1}$. Therefore $x^{k-1}<_{b} y^{k}<_{b} x^{k}$, which implies $x(b)=b$. By equation

$$
r_{5}=\left(w^{-k} x^{k}\right)^{l}\left(y^{-k} x^{k}\right)^{l-1}\left(y^{-k} x^{k-1}\right)=1
$$

we obtain that $\left(w^{-k} x^{k}\right)^{l}(b)=b$, which implies $\left(w^{-k} x^{k}\right)(b)=b$. Hence $w^{-k}(b)=b$, and $w(b)=b$. This is impossible because $w^{-1}(b)=b$ and $w^{-1}>1$ implies that $1<_{b} w^{-1}$, which is a contradiction to the fact that $1<_{b} w$. Therefore case (3) is not possible.

For (4), Lemma 3.3 implies $x^{-k} y^{k}(b) \leq b, x^{k} y^{-k}(b) \leq b$, hence $x^{-k}(b) \geq y^{-k}(b)=b$ and $b=y^{k}(b) \leq x^{k}(b)$. This two inequalities imply that $x(b)=b$. Therefore, by relation

$$
r_{5}=\left(w^{-k} x^{k}\right)^{l}\left(y^{-k} x^{k}\right)^{l-1}\left(y^{-k} x^{k-1}\right)=1
$$

we obtain that $\left(w^{-k} x^{k}\right)^{l}(b)=b$, which implies $\left(w^{-k} x^{k}\right)(b)=b$. Hence $w^{-k}(b)=b$, and $w(b)=b$. A similar argument using relation $r_{4}$ shows that $t(b)=b$. Since $x y z t w=1, z(b)=b$. Thus $b$ is fixed by $G$, which contradicts our assumptions. Therefore case (4) is not possible.

Lemma 3.10. Let $c$ be a fixed point for $z$. Then the only possible signs for $x, y, z, t, w$ with respect to $<_{c}$ are $1<_{c} x, 1<_{c} y, z<_{c} 1, t<_{c} 1, w<_{c} 1$.

Proof. Since $z<1$ and $z(c)=c$, we have $z<_{c} 1$. Let $<^{\prime}=<_{c}^{o p}$, then $1<^{\prime} z$. Therefore as in the analysis of (1) through (16) above, by replacing $x$ by $z, y$ by $t, z$ by $w, t$ by $x, w$ by $y$ and $<$ by $<^{\prime}$, one can see that the cases which are left to check are 
(1) $1<^{\prime} z, 1<^{\prime} t, w<^{\prime} 1, x<^{\prime} 1, y<^{\prime} 1$

(2) $t<^{\prime} 1, w<^{\prime} 1,1<^{\prime} x, 1<^{\prime} y, 1<^{\prime} z$

(3) $w<^{\prime} 1, x<^{\prime} 1,1<^{\prime} y, 1<^{\prime} z, 1<^{\prime} t$

(4) $x<^{\prime} 1, y<^{\prime} 1,1<^{\prime} z, 1<^{\prime} t, 1<^{\prime} w$

(5) $1<^{\prime} y, 1<^{\prime} z, t<^{\prime} 1, w<^{\prime} 1, x<^{\prime} 1$

Therefore,

(1) $z<_{c} 1, t<_{c} 1,1<_{c} w, 1<_{c} x, 1<_{c} y$

(2) $1<_{c} t, 1<_{c} w, x<_{c} 1, y<_{c} 1, z<_{c} 1$

(3) $1<_{c} w, 1<_{c} x, y<_{c} 1, z<_{c} 1, t<_{c} 1$

(4) $1<_{c} x, 1<_{c} y, z<_{c} 1, t<_{c} 1, w<_{c} 1$

(5) $y<_{c} 1, z<_{c} 1,1<_{c} t, 1<_{c} w, 1<_{c} x$

For (1), let $<^{\prime}=<_{c}^{o p}$, using Lemma 3.3 and replacing $x$ by $z, y$ by $t, z$ by $w, t$ by $x$ and $w$ by $y$, we have $t^{k}<^{\prime} z^{k}, t^{-k}<^{\prime} z^{-k}$. Therefore $z^{k}<_{c} t^{k}, z^{-k}<_{c} t^{-k}$, which implies $t(c)=c$, using equation $r_{2}$ we have that $y(c)=c$ and using equation $r_{1}$ that $x(c)=c$, and therefore by equation $r_{5}$, we have that $w(c)=c$, which is impossible because $w^{-1}(c)=c$ and $w^{-1}>1$ implies that $1<_{c} w^{-1}$, which is a contradiction to the fact that $1<_{c} w$. Therefore case (1) is not possible.

For (2), using Lemma 3.5 and replacing $x$ by $t, y$ by $w, z$ by $x, t$ by $y$ and $w$ by $z$ we have $y^{k}<_{c} z^{k}<_{c} y^{k-1}$, which implies $y(c)=c$. This is impossible because $y(c)=c$ and $y>1$ implies that $1<_{c} y$, which is a contradiction to the fact that $y<_{c} 1$. Therefore case (2) is not possible.

For (3), using Lemma 3.5 and replacing $x$ by $w, y$ by $x, z$ by $y, t$ by $z$ and $w$ by $t$, we have $z^{k}<_{c} t^{k}<_{c} z^{k-1}$, which implies $t(c)=c$, using equation $r_{2}$ we have that $y(c)=c$, which is impossible because $y(c)=c$ and $y>1$ implies that $1<_{c} y$, which is a contradiction to the fact that $y<_{c} 1$. Therefore case (3) is not possible.

For (5), let $<^{\prime}=<_{c}^{o p}$, using Lemma 3.3, and replacing $x$ by $y, y$ by $z, z$ by $t, t$ by $w$ and $w$ by $x$, we have $z^{k}<^{\prime} y^{k}, z^{-k}<^{\prime} y^{-k}$. Therefore $y^{k}<_{c} z^{k}, y^{-k}<_{c} z^{-k}$, which implies $y(c)=c$. This is impossible because $y(c)=c$ and $y>1$ implies that $1<_{c} y$, which contradicts the fact that $y<_{c} 1$. Therefore case $(5)$ is not possible.

Lemma 3.11. Let $d$ be a fixed point for $t$. Then the only possible signs for $x, y, z, t, w$ with respect to $<_{d}$ are $1<_{d} y, 1<_{d} z, t<_{d} 1, w<_{d} 1, x<_{d} 1$.

Proof. Similar proof as for Lemma 3.8.

Lemma 3.12. Let $e$ be a fixed point for $w$. Then the only possible signs for $x, y, z, t, w$ with respect to $<_{e}$ are $1<_{e} z, 1<_{e} t, w<_{e} 1, x<_{e} 1, y<_{e} 1$.

Proof. Similar proof as for Lemma 3.8. 
Summarizing Lemmas 3.8 through 3.12, we have

\section{Remark 3.13.}

(1) If $e$ is a fixed point for $w$, then the only possible signs for $x, y, z, t, w$ with respect to $<_{e}$ are $1<_{e} z, 1<_{e} t, w<_{e} 1, x<_{e} 1, y<_{e} 1$. Therefore $x, y, z, t$ will also have fixed points (different to $e$ ).

(2) If $d$ is a fixed point for $t$, then the only possible signs for $x, y, z, t, w$ with respect to $<_{d}$ are $1<_{d} y, 1<_{d} z, t<_{d} 1, w<_{d} 1, x<_{d} 1$. Therefore $x, z$ will also have fixed points (different to $d)$.

(3) If $b$ is a fixed point for $y$, then the only possible signs for $x, y, z, t, w$ with respect to $<_{b}$ are $w<_{b} 1, x<_{b} 1,1<_{b} y, 1<_{b} z, 1<_{b} t$. Therefore $x, z, t$ will also have fixed points (different to $b)$.

(4) If $a$ is a fixed point for $x$, then the only possible signs for $x, y, z, t, w$ with respect to $<_{a}$ are $t<_{a} 1, w<_{a} 1,1<_{a} x, 1<_{a} y, 1<_{a} z$. Therefore $z$ also has a fixed point (different to $a$ ).

Lemma 3.14. $z$ has a fixed point.

Proof. Assume by contradiction that $z$ is fixed point free. Then by the previous remark, $x$, $y, t, w$ are also fixed point free. Therefore, for any $a \in \mathbb{R}$ the only possible signs for $x, y, z$, $t, w$ with respect to $<_{a}$ are $1<_{a} x, 1<_{a} y, z<_{a} 1, t<_{a} 1, w<_{a} 1$. Then by Lemma 3.3, $x^{-k} y^{k}(a) \leq a, x^{k} y^{-k}(a) \leq a$, which implies $y^{k}(a) \leq x^{k}(a)$ and $y^{-k}(a) \leq x^{-k}(a)$. Since $a \in \mathbb{R}$ was arbitrary, the last two inequalities are true for any $a \in \mathbb{R}$. Then $a \leq y^{-k}\left(x^{k}(a)\right)$ for any $a \in \mathbb{R}$, which implies $a \leq y^{-k}\left(x^{k}(a)\right) \leq x^{-k}\left(x^{k}(a)\right)=a$, hence $x^{k}(a)=y^{k}(a)$ for any $a \in \mathbb{R}$. Therefore $x^{k}=y^{k}$. We obtain a contradiction from the equation

$$
r_{1}=\left(x^{-k} y^{k}\right)^{l}\left(z^{-k} y^{k}\right)^{l-1}\left(z^{-k} y^{k-1}\right)=1
$$

since $y>1, z^{-1}>1$ and $x^{-k} y^{k}=1$.

Proof of Theorem 1.3. The equation

$$
r_{3}=\left(z^{-k} t^{k}\right)^{l}\left(w^{-k} t^{k}\right)^{l-1}\left(w^{-k} t^{k-1}\right)=1
$$

implies

$$
z^{-k}\left(t^{k} z^{-k}\right)^{l-1}\left(t^{k} w^{-k}\right)^{l} t^{k-1}=1
$$

which implies

$$
t^{k-1} z^{-k}\left(t^{k} z^{-k}\right)^{l-1}\left(t^{k} w^{-k}\right)^{l}=1
$$

Since $t^{k} z^{-k}<1$ by Lemma $3.5, t^{k} w^{-k}<1$ by Lemma 3.6, and $t^{k-1} z^{-k}\left(t^{k} z^{-k}\right)^{l-1}\left(t^{k} w^{-k}\right)^{l}=1$, then $t^{k-1} z^{-k} \geq 1$.

By Lemma $3.14 z$ has a fixed point, call it $c$. By Lemma 3.10, the only possible signs for $x$, $y, z, t, w$ with respect to $<_{c}$ are $1<_{c} x, 1<_{c} y, z<_{c} 1, t<_{c} 1, w<_{c} 1$. Then $1 \leq_{c} t^{k-1} z^{-k}$. This implies that $t^{-k+1}(c) \leq z^{-k}(c)=c$. Hence $t^{-k+1}(c)=c$, which implies $t(c)=c$, and this is impossible by Lemma 3.11. 


\section{LEFT-ORDERABILITY AND GENUS 2 TWO-BRIDGE KNOTS}

In this section we will prove the following theorem.

Theorem 4.1. The fundamental group of the 3-fold cyclic branched cover of $K_{[-2 q, 2 s,-2 t, 2 l]}$ is not left-orderable where $q, s, t$ and $l \in \mathbb{Z} \backslash\{0\}$.

Mulazzani and Vesnin ([MV], Theorem 8) proved that the generalized periodic Takahashi manifold $T_{n, m}\left(\frac{1}{a_{j}} ; \frac{1}{b_{j}}\right)$ is the $n$-fold cyclic branched covering of the two-bridge knot corresponding to the Conway parameters $\left[-2 a_{1}, 2 b_{1},-2 a_{2}, 2 b_{2}, \cdots,-2 a_{m}, 2 b_{m}\right]$. For $m=2$ we have the family of genus 2 two-bridge knots $K_{[-2 q, 2 s,-2 t, 2 l]}$. In order to show that the fundamental group of the 3 -fold cyclic branched cover of any genus 2 two-bridge knot $K_{[-2 q, 2 s,-2 t, 2 l]}$ is not left-orderable we have to consider all cases for the signs of $q, s, t$ and $l$. We have sixteen cases to consider, but since the mirror image of the knot $K_{[-2 q, 2 s,-2 t, 2 l]}$ is the knot $K_{[2 q,-2 s, 2 t,-2 l]}$ we need only deal with eight of them:

(1) $q>0, s>0, t>0$ and $l>0$

(2) $q>0, s>0, t<0$ and $l>0$;

(3) $q<0, s>0, t<0$ and $l>0$;

(4) $q<0, s<0, t<0$ and $l>0$;

(5) $q<0, s>0, t>0$ and $l>0$;

(6) $q<0, s>0, t<0$ and $l<0$;

(7) $q>0, s<0, t<0$ and $l>0$;

(8) $q>0, s>0, t<0$ and $l<0$.

By Mulazzani and Vesnin ([MV], Theorem 10), the fundamental group of the $n$-fold cyclic branched cover of $K_{[-2 q, 2 s,-2 t, 2 l]}$ is

$$
G:=\pi_{1}\left(\Sigma_{n}\left(K_{[-2 q, 2 s,-2 t, 2 l]}\right)\right)=\left\langle x_{1}, x_{2}, \cdots, x_{n} \mid w\left(x_{k-2}, x_{k-1}, x_{k}, x_{k+1}, x_{k+2}\right), k \in \mathbb{Z} / n\right\rangle
$$

where

$w\left(x_{k-2}, x_{k-1}, x_{k}, x_{k+1}, x_{k+2}\right)=$

$\left[\left[\left(x_{k}^{q} x_{k+1}^{-q}\right)^{-s} x_{k}\left(x_{k-1}^{q} x_{k}^{-q}\right)^{s}\right]^{t} x_{k}^{q} x_{k+1}^{-q}\left[\left(x_{k+1}^{q} x_{k+2}^{-q}\right)^{-s} x_{k+1}\left(x_{k}^{q} x_{k+1}^{-q}\right)^{s}\right]^{-t}\right]^{-l}\left(x_{k}^{q} x_{k+1}^{-q}\right)^{-s} x_{k}\left(x_{k-1}^{q} x_{k}^{-q}\right)^{s}$ $\cdot\left[\left[\left(x_{k-1}^{q} x_{k}^{-q}\right)^{-s} x_{k-1}\left(x_{k-2}^{q} x_{k-1}^{-q}\right)^{s}\right]^{t} x_{k-1}^{q} x_{k}^{-q}\left[\left(x_{k}^{q} x_{k+1}^{-q}\right)^{-s} x_{k}\left(x_{k-1}^{q} x_{k}^{-q}\right)^{s}\right]^{-t}\right]^{l}$ and $k \in \mathbb{Z} / n$.

Since there is an automorphism of $G$ given by sending $x_{k}$ to $x_{k+1}$, for each $k \in \mathbb{Z} / n$ and $K_{[-2 q, 2 s,-2 t, 2 l]}$ is nontrivial no $x_{k}$ is trivial.

For $n=3$, let $x=x_{1}, y=x_{2}$ and $z=x_{3}$, we have

$$
\begin{aligned}
& r_{1}=\left[\left[\left(y^{q} x^{-q}\right)^{s} x\left(z^{q} x^{-q}\right)^{s}\right]^{t} x^{q} y^{-q}\left[\left(z^{q} y^{-q}\right)^{s} y\left(x^{q} y^{-q}\right)^{s}\right]^{-t}\right]^{-l}\left(y^{q} x^{-q}\right)^{s} x\left(z^{q} x^{-q}\right)^{s} \\
& \cdot\left[\left[\left(x^{q} z^{-q}\right)^{s} z\left(y^{q} z^{-q}\right)^{s}\right]^{t} z^{q} x^{-q}\left[\left(y^{q} x^{-q}\right)^{s} x\left(z^{q} x^{-q}\right)^{s}\right]^{-t}\right]^{l}=1 ; \\
& r_{2}=\left[\left[\left(z^{q} y^{-q}\right)^{s} y\left(x^{q} y^{-q}\right)^{s}\right]^{t} y^{q} z^{-q}\left[\left(x^{q} z^{-q}\right)^{s} z\left(y^{q} z^{-q}\right)^{s}\right]^{-t}\right]^{-l}\left(z^{q} y^{-q}\right)^{s} y\left(x^{q} y^{-q}\right)^{s} \\
& \cdot\left[\left[\left(y^{q} x^{-q}\right)^{s} x\left(z^{q} x^{-q}\right)^{s}\right]^{t} x^{q} y^{-q}\left[\left(z^{q} y^{-q}\right)^{s} y\left(x^{q} y^{-q}\right)^{s}\right]^{-t}\right]^{l}=1 ;
\end{aligned}
$$


$r_{3}=\left[\left[\left(x^{q} z^{-q}\right)^{s} z\left(y^{q} z^{-q}\right)^{s}\right]^{t} z^{q} x^{-q}\left[\left(y^{q} x^{-q}\right)^{s} x\left(z^{q} x^{-q}\right)^{s}\right]^{-t}\right]^{-l}\left(x^{q} z^{-q}\right)^{s} z\left(y^{q} z^{-q}\right)^{s}$

$\cdot\left[\left[\left(z^{q} y^{-q}\right)^{s} y\left(x^{q} y^{-q}\right)^{s}\right]^{t} y^{q} z^{-q}\left[\left(x^{q} z^{-q}\right)^{s} z\left(y^{q} z^{-q}\right)^{s}\right]^{-t}\right]^{l}=1$.

Proof of Theorem 4.1. Considering the product $r_{3} r_{2} r_{1}$ we have,

$$
z y x=1
$$

We prove Theorem 4.1 by contradiction. Let $<$ be a left-order on $G$. Without loss of generality $x>1$. Here is the list of possible signs for $y, z$.

(1) $x>1, y>1, z>1$

(2) $x>1, y<1, z>1$

(3) $x>1, y<1, z<1$;

(4) $x>1, y>1, z<1$;

We can immediately rule out (1) because $z y x=1$. The cases which are left to check are (2), (3), (4).

The automorphism $\phi$ of $G$ which sends $(x, y, z)$ to $(z, x, y)$ acts on $L O(G)$. Using $\phi$ the reader will verify that up to replacing an order by its opposite, an order of the form (2), (3), (4), exists if and only if one of type $x>1, y<1, z<1$ exists. Assume that $<$ satisfies these inequalities.

Let

$$
\begin{aligned}
& X=\left(y^{q} x^{-q}\right)^{s} x\left(z^{q} x^{-q}\right)^{s} \\
& Y=\left(z^{q} y^{-q}\right)^{s} y\left(x^{q} y^{-q}\right)^{s}
\end{aligned}
$$

and

$$
Z=\left(x^{q} z^{-q}\right)^{s} z\left(y^{q} z^{-q}\right)^{s} .
$$

Considering the product $r_{3} r_{2} r_{1}$ we have, $Z Y X=1$ and $r_{1}, r_{2}$, and $r_{3}$ become,

- $r_{1}^{\prime}=\left(Y^{t} y^{q} x^{-q} X^{-t}\right)^{l} X\left(Z^{t} z^{q} x^{-q} X^{-t}\right)^{l}=1$;

- $r_{2}^{\prime}=\left(Z^{t} z^{q} y^{-q} Y^{-t}\right)^{l} Y\left(X^{t} x^{q} y^{-q} Y^{-t}\right)^{l}=1$;

- $r_{3}^{\prime}=\left(X^{t} x^{q} z^{-q} Z^{-t}\right)^{l} Z\left(Y^{t} y^{q} z^{-q} Z^{-t}\right)^{l}=1$.

This is the same as,

- $r_{1}^{\prime \prime}=\left(Y^{t} y^{q} x^{-q} X^{-t}\right)^{l-1} Y^{t} y^{q} x^{-q} X^{-t+1}\left(Z^{t} z^{q} x^{-q} X^{-t}\right)^{l}=1$;

- $r_{2}^{\prime \prime}=\left(Z^{t} z^{q} y^{-q} Y^{-t}\right)^{l-1} Z^{t} z^{q} y^{-q} Y^{-t+1}\left(X^{t} x^{q} y^{-q} Y^{-t}\right)^{l}=1$;

- $r_{3}^{\prime \prime}=\left(X^{t} x^{q} z^{-q} Z^{-t}\right)^{l-1} X^{t} x^{q} z^{-q} Z^{-t+1}\left(Y^{t} y^{q} z^{-q} Z^{-t}\right)^{l}=1$.

We have eight cases:

First case: $q>0, s>0, t>0, l>0$.

We have $X=\left(y^{q} x^{-q}\right)^{s} x\left(z^{q} x^{-q}\right)^{s}=\left(y^{q} x^{-q}\right)^{s-1} y^{q} x^{-q+1}\left(z^{q} x^{-q}\right)^{s}$. Since $y<1, z<1$ and $x^{-1}<1$ then $X<1$.

We discuss the signs of $Y$ and $Z$. We have three subcases 
(1) If $Y<1$, then since $Z Y X=1$ and $X<1$ then $Z>1$. Since $Y=\left(z^{q} y^{-q}\right)^{s} y\left(x^{q} y^{-q}\right)^{s}=$ $\left(z^{q} y^{-q}\right)^{s} y x x^{q-1} y^{-q}\left(x^{q} y^{-q}\right)^{s-1}=\left(z^{q} y^{-q}\right)^{s} z^{-1} x^{q-1} y^{-q}\left(x^{q} y^{-q}\right)^{s-1}$ then $z^{q} y^{-q}<1$. We have,

$$
\begin{aligned}
X^{t} x^{q} z^{-q} & =X^{t-1}\left(y^{q} x^{-q}\right)^{s} x\left(z^{q} x^{-q}\right)^{s} x^{q} z^{-q} \\
& =X^{t-1}\left(y^{q} x^{-q}\right)^{s} x\left(z^{q} x^{-q}\right)^{s-1} z^{q} x^{-q} x^{q} z^{-q} \\
& =X^{t-1}\left(y^{q} x^{-q}\right)^{s} x\left(z^{q} x^{-q}\right)^{s-1} \\
& =X^{t-1}\left(y^{q} x^{-q}\right)^{s-1} y^{q} x^{-q+1}\left(z^{q} x^{-q}\right)^{s-1}
\end{aligned}
$$

Since $X<1, y<1, z<1$ and $x^{-1}<1$ then $X^{t} x^{q} z^{-q}<1$. Therefore $X^{t} x^{q} z^{-q} Z^{-t}<1$ and $X^{t} x^{q} z^{-q} Z^{-t+1}<1$.

We have also that,

$$
\begin{aligned}
y^{q} z^{-q} Z^{-t} & =y^{q} z^{-q}\left(z^{q} y^{-q}\right)^{s} z^{-1}\left(z^{q} x^{-q}\right)^{s} Z^{-t+1} \\
& =y^{q} z^{-q} z^{q} y^{-q}\left(z^{q} y^{-q}\right)^{s-1} z^{-1}\left(z^{q} x^{-q}\right)^{s} Z^{-t+1} \\
& =\left(z^{q} y^{-q}\right)^{s-1} z^{q-1} x^{-q}\left(z^{q} x^{-q}\right)^{s-1} Z^{-t+1}
\end{aligned}
$$

Since $Z^{-1}<1, z^{q} y^{-q}<1, z<1$ and $x^{-1}<1$ then $y^{q} z^{-q} Z^{-t}<1$. Therefore $Y^{t} y^{q} z^{-q} Z^{-t}<1$, and we have a contradiction by the relation $r_{3}^{\prime \prime}$.

(2) If $Z<1$ then since $Z Y X=1$ and $X<1$ then $Y>1$. Since $Z=\left(x^{q} z^{-q}\right)^{s} z\left(y^{q} z^{-q}\right)^{s}=$ $\left(x^{q} z^{-q}\right)^{s-1} x^{q} z^{-q+1}\left(y^{q} z^{-q}\right)^{s}$ then $y^{q} z^{-q}<1$. We have

$$
\begin{aligned}
z^{q} y^{-q} Y^{-t} & =z^{q} y^{-q}\left(y^{q} x^{-q}\right)^{s} y^{-1}\left(y^{q} z^{-q}\right)^{s} Y^{-t+1} \\
& =z^{q} y^{-q} y^{q} x^{-q}\left(y^{q} x^{-q}\right)^{s-1} y^{-1}\left(y^{q} z^{-q}\right)^{s} Y^{-t+1} \\
& =z^{q} x^{-q}\left(y^{q} x^{-q}\right)^{s-1} y^{-1}\left(y^{q} z^{-q}\right)^{s} Y^{-t+1} \\
& =z^{q} x^{-q}\left(y^{q} x^{-q}\right)^{s-2} y^{q} x^{-q+1} x^{-1} y^{-1}\left(y^{q} z^{-q}\right)^{s} Y^{-t+1} \\
& =z^{q} x^{-q}\left(y^{q} x^{-q}\right)^{s-2} y^{q} x^{-q+1} z\left(y^{q} z^{-q}\right)^{s} Y^{-t+1}
\end{aligned}
$$

Since $Y^{-1}<1$ and $y^{q} z^{-q}<1$ then $z^{q} y^{-q} Y^{-t}<1$. Therefore $Z^{t} z^{q} y^{-q} Y^{-t}<1$ and $Z^{t} z^{q} y^{-q} Y^{-t+1}<1$ for $t>1$. For $t=1$, the relation $r_{2}^{\prime \prime}$ become

$$
r_{2}^{\prime \prime \prime}=\left(Z z^{q} y^{-q} Y^{-1}\right)^{l-1} Z z^{q} y^{-q} X x^{q} y^{-q} Y^{-1}\left(X x^{q} y^{-q} Y^{-1}\right)^{l-1}=1
$$

and we have

$$
z^{q} y^{-q} X=z^{q} y^{-q} y^{q} x^{-q}\left(y^{q} x^{-q}\right)^{s-1} x\left(z^{q} x^{-q}\right)^{s}=z^{q} x^{-q}\left(y^{q} x^{-q}\right)^{s-2} y^{q} x^{-q+1}\left(z^{q} x^{-q}\right)^{s}<1 .
$$

We have also that,

$$
\begin{aligned}
Y^{t} y^{q} x^{-q} & =Y^{t-1}\left(z^{q} y^{-q}\right)^{s} y\left(x^{q} y^{-q}\right)^{s} y^{q} x^{-q} \\
& =Y^{t-1}\left(z^{q} y^{-q}\right)^{s} y\left(x^{q} y^{-q}\right)^{s-1} x^{q} y^{-q} y^{q} x^{-q} \\
& =Y^{t-1}\left(z^{q} y^{-q}\right)^{s} y\left(x^{q} y^{-q}\right)^{s-1}=Y^{t-1}\left(z^{q} y^{-q}\right)^{s} y x x^{q-1} y^{-q}\left(x^{q} y^{-q}\right)^{s-2} \\
& =Y^{t-1}\left(z^{q} y^{-q}\right)^{s} z^{-1} x^{q-1} y^{-q}\left(x^{q} y^{-q}\right)^{s-2}
\end{aligned}
$$

Since $z^{q} y^{-q}>1, z^{-1}>1$ and $y^{-1}>1$ then $Y^{t} y^{q} x^{-q}>1$. Hence $Y^{t} y^{q} x^{-q} X^{-t}>1$ which implies $X^{t} x^{q} y^{-q} Y^{-t}=\left(Y^{t} y^{q} x^{-q} X^{-t}\right)^{-1}<1$. Therefore by relation $r_{2}^{\prime \prime}$ and $r_{2}^{\prime \prime \prime}$ we have a contradiction. 
(3) The only subcase which is left to check is if $Y>1$ and $Z>1$. In this subcase we have two subsubcases

- If $z^{q} y^{-q}>1$ then by the last part of subcase (2) we have $Y^{t} y^{q} x^{-q} X^{-t}>1$, $Y^{t} y^{q} x^{-q} X^{-t+1}>1$ and by the first part of subcase (1) we have that $X^{t} x^{q} z^{-q} Z^{-t}<$ 1 which implies $Z^{t} z^{q} x^{-q} X^{-t}=\left(X^{t} x^{q} z^{-q} Z^{-t}\right)^{-1}>1$. This gives a contradiction by the relation $r_{1}^{\prime \prime}$.

- If $z^{q} y^{-q}<1$ then $y^{q} z^{-q}>1$. We have

$$
\begin{aligned}
y^{q} x^{-q} X^{-t} & =y^{q} x^{-q}\left(x^{q} z^{-q}\right)^{s} x^{-1}\left(x^{q} y^{-q}\right)^{s} X^{-t+1} \\
& =y^{q} x^{-q} x^{q} z^{-q}\left(x^{q} z^{-q}\right)^{s-1} x^{-1}\left(x^{q} y^{-q}\right)^{s} X^{-t+1} \\
& =y^{q} z^{-q}\left(x^{q} z^{-q}\right)^{s-1} x^{-1}\left(x^{q} y^{-q}\right)^{s} X^{-t+1} \\
& =y^{q} z^{-q}\left(x^{q} z^{-q}\right)^{s-1} x^{q-1} y^{-q}\left(x^{q} y^{-q}\right)^{s-1} X^{-t+1}
\end{aligned}
$$

Since $X^{-1}>1, y^{q} z^{-q}>1 z^{-1}>1$ and $y^{-1}>1$ then $y^{q} x^{-q} X^{-t}>1$. Hence $Y^{t} y^{q} x^{-q} X^{-t}>1$ and $Y^{t} y^{q} x^{-q} X^{-t+1}>1$ if $t>1$. If $t=1$, then relation $r_{1}^{\prime \prime}$ become

$$
r_{1}^{\prime \prime \prime}=\left(Y y^{q} x^{-q} X^{-1}\right)^{l-1} Y y^{q} x^{-q} Z z^{q} x^{-q} X^{-1}\left(Z z^{q} x^{-q} X^{-1}\right)^{l-1}=1
$$

and we have

$y^{q} x^{-q} Z=y^{q} x^{-q} x^{q} z^{-q}\left(x^{q} z^{-q}\right)^{s-1} z\left(y^{q} z^{-q}\right)^{s}=y^{q} z^{-q}\left(x^{q} z^{-q}\right)^{s-2} x^{q} z^{-q+1}\left(y^{q} z^{-q}\right)^{s}>$ 1. We have also by the first part of subcase (1) we have that $X^{t} x^{q} z^{-q} Z^{-t}<1$ which implies $Z^{t} z^{q} x^{-q} X^{-t}=\left(X^{t} x^{q} z^{-q} Z^{-t}\right)^{-1}>1$. This will gives a contradiction by the relations $r_{1}^{\prime \prime}$ and $r_{1}^{\prime \prime \prime}$.

Second case: $q>0, s>0, t<0, l>0$.

We have $X=\left(y^{q} x^{-q}\right)^{s} x\left(z^{q} x^{-q}\right)^{s}=\left(y^{q} x^{-q}\right)^{s-1} y^{q} x^{-q+1}\left(z^{q} x^{-q}\right)^{s}$. Since $y<1, z<1$ and $x^{-1}<1$ then $X<1$.

Similarly as the first case we have three subcases:

(1) If $Y<1$, then since $Z Y X=1$ and $X<1$ then $Z>1$. Since $Y=\left(z^{q} y^{-q}\right)^{s} y\left(x^{q} y^{-q}\right)^{s}=$ $\left(z^{q} y^{-q}\right)^{s} y x x^{q-1} y^{-q}\left(x^{q} y^{-q}\right)^{s-1}=\left(z^{q} y^{-q}\right)^{s} z^{-1} x^{q-1} y^{-q}\left(x^{q} y^{-q}\right)^{s-1}$ then $z^{q} y^{-q}<1$ which implies $y^{q} z^{-q}>1$. By the relation

$$
r_{3}^{\prime}=\left(X^{t} x^{q} z^{-q} Z^{-t}\right)^{l} Z\left(Y^{t} y^{q} z^{-q} Z^{-t}\right)^{l}=1
$$

we have a contradiction.

(2) If $Z<1$ then since $Z Y X=1$ and $X<1$ then $Y>1$. Since $Z=\left(x^{q} z^{-q}\right)^{s} z\left(y^{q} z^{-q}\right)^{s}=$ $\left(x^{q} z^{-q}\right)^{s-1} x^{q} z^{-q+1}\left(y^{q} z^{-q}\right)^{s}$ then $y^{q} z^{-q}<1$ which implies $z^{q} y^{-q}>1$. By relation

$$
r_{2}^{\prime}=\left(Z^{t} z^{q} y^{-q} Y^{-t}\right)^{l} Y\left(X^{t} x^{q} y^{-q} Y^{-t}\right)^{l}=1
$$

we have a contradiction.

(3) The only subcase which is left to check is if $Y>1$ and $Z>1$. In this subcase the relation

$$
r_{1}^{\prime}=\left(Y^{t} y^{q} x^{-q} X^{-t}\right)^{l} X\left(Z^{t} z^{q} x^{-q} X^{-t}\right)^{l}=1
$$

gives a contradiction. 
Third case: $q<0, s>0, t<0$ and $l>0$.

We have $X=\left(y^{q} x^{-q}\right)^{s} x\left(z^{q} x^{-q}\right)^{s}>1$. Similarly as the first case we have three subcases:

(1) If $Y>1$, then since $Z Y X=1$ and $X>1$ then $Z<1$. Since $Y=\left(z^{q} y^{-q}\right)^{s} y\left(x^{q} y^{-q}\right)^{s}$ then $z^{q} y^{-q}>1$ which implies that $y^{q} z^{-q}<1$. Therefore by the relation

$$
r_{3}^{\prime}=\left(X^{t} x^{q} z^{-q} Z^{-t}\right)^{l} Z\left(Y^{t} y^{q} z^{-q} Z^{-t}\right)^{l}=1
$$

we have a contradiction.

(2) If $Z>1$, then since $Z Y X=1$ and $X>1$ then $Y<1$. Since $Z=\left(x^{q} z^{-q}\right)^{s} z\left(y^{q} z^{-q}\right)^{s}$ then $y^{q} z^{-q}>1$ which implies that $z^{q} y^{-q}<1$. Therefore we have a contradiction by the relation

$$
r_{2}^{\prime}=\left(Z^{t} z^{q} y^{-q} Y^{-t}\right)^{l} Y\left(X^{t} x^{q} y^{-q} Y^{-t}\right)^{l}=1
$$

(3) The only subcase which is left to check is if $Y<1$ and $Z<1$. In this subcase the relation

$$
r_{1}^{\prime}=\left(Y^{t} y^{q} x^{-q} X^{-t}\right)^{l} X\left(Z^{t} z^{q} x^{-q} X^{-t}\right)^{l}=1
$$

gives a contradiction.

Fourth case: $q<0, s<0, t<0$ and $l>0$.

We have $X=\left(x^{q} y^{-q}\right)^{-s} x\left(x^{q} z^{-q}\right)^{-s}=\left(x^{q} y^{-q}\right)^{-s} x^{q+1} z^{-q}\left(x^{q} z^{-q}\right)^{-s-1}<1$. Similarly as the first case we have three subcases:

(1) If $Y<1$, then since $Z Y X=1$ and $X<1$ then $Z>1$. Since $Y=\left(y^{q} z^{-q}\right)^{-s} y\left(y^{q} x^{-q}\right)^{-s}=$ $\left(y^{q} z^{-q}\right)^{-s} y^{q+1} x^{-q}\left(y^{q} x^{-q}\right)^{-s-1}$ then $y^{q} z^{-q}<1$, so $z^{q} y^{-q}>1$. We have

$$
\begin{aligned}
Z^{t} z^{q} y^{-q} & =Z^{t+1}\left(y^{q} z^{-q}\right)^{-s} z^{-1}\left(x^{q} z^{-q}\right)^{-s} z^{q} y^{-q} \\
& =Z^{t+1}\left(y^{q} z^{-q}\right)^{-s} z^{-1}\left(x^{q} z^{-q}\right)^{-s-1} x^{q} z^{-q} z^{q} y^{-q} \\
& =Z^{t+1}\left(y^{q} z^{-q}\right)^{-s} z^{-1} x^{q} z^{-q}\left(x^{q} z^{-q}\right)^{-s-2} x^{q} y^{-q} \\
& =Z^{t+1}\left(y^{q} z^{-q}\right)^{-s} z^{-1} x^{-1} x^{q+1} z^{-q}\left(x^{q} z^{-q}\right)^{-s-2} x^{q} y^{-q} \\
& =Z^{t+1}\left(y^{q} z^{-q}\right)^{-s} y x^{q+1} z^{-q}\left(x^{q} z^{-q}\right)^{-s-2} x^{q} y^{-q}>1 .
\end{aligned}
$$

We have also that

$$
\begin{aligned}
Z^{t} z^{q} x^{-q} & =Z^{t+1}\left(y^{q} z^{-q}\right)^{-s} z^{-1}\left(x^{q} z^{-q}\right)^{-s} z^{q} x^{-q} \\
& =Z^{t+1}\left(y^{q} z^{-q}\right)^{-s} z^{-1}\left(x^{q} z^{-q}\right)^{-s-1} x^{q} z^{-q} z^{q} x^{-q} \\
& =Z^{t+1}\left(y^{q} z^{-q}\right)^{-s} z^{-1}\left(x^{q} z^{-q}\right)^{-s-1} \\
& =Z^{t+1}\left(y^{q} z^{-q}\right)^{-s} z^{-1} x^{q} z^{-q}\left(x^{q} z^{-q}\right)^{-s-2} \\
& =Z^{t+1}\left(y^{q} z^{-q}\right)^{-s} z^{-1} x^{-1} x^{q+1} z^{-q}\left(x^{q} z^{-q}\right)^{-s-2} \\
& =Z^{t+1}\left(y^{q} z^{-q}\right)^{-s} y x^{q+1} z^{-q}\left(x^{q} z^{-q}\right)^{-s-2}<1, \text { if } s<-1 .
\end{aligned}
$$


Therefore $x^{q} z^{-q} Z^{-t}>1$, and $X^{t} x^{q} z^{-q} Z^{-t}>1$ if $s<-1$. If $s=-1$ then

$$
\begin{aligned}
Z^{t} z^{q} x^{-q} X^{-t} & =Z^{t+1}\left(y^{q} z^{-q}\right) z^{-1} X X^{-t-1} \\
& =Z^{t+1}\left(y^{q} z^{-q}\right) z^{-1} x^{q} y^{-q} x x^{q} z^{-q} X^{-t-1} \\
& =Z^{t+1}\left(y^{q} z^{-q}\right) z^{-1} x^{-1} x^{q+1} y^{-q} x^{q+1} z^{-q} X^{-t-1} \\
& =Z^{t+1}\left(y^{q} z^{-q}\right) y x^{q+1} y^{-q} x^{q+1} z^{-q} X^{-t-1}<1
\end{aligned}
$$

Hence $X^{t} x^{q} z^{-q} Z^{-t}>1$ if $s=-1$. Therefore by $r_{3}^{\prime}$ we have a contradiction.

(2) If $Z<1$ then since $Z Y X=1$ and $X<1$ then $Y>1$. Since

$$
\begin{aligned}
Z & =\left(z^{q} x^{-q}\right)^{-s} z\left(z^{q} y^{-q}\right)^{-s} \\
& =\left(z^{q} x^{-q}\right)^{-s-1} z^{q} x^{-q} z\left(z^{q} y^{-q}\right)^{-s} \\
& =\left(z^{q} x^{-q}\right)^{-s-1} z^{q} x^{-q-1} x z\left(z^{q} y^{-q}\right)^{-s} \\
& =\left(z^{q} x^{-q}\right)^{-s-1} z^{q} x^{-q-1} y^{-1}\left(z^{q} y^{-q}\right)^{-s}
\end{aligned}
$$

then $z^{q} y^{-q}<1$.

We have,

$$
\begin{aligned}
Y^{t} y^{q} z^{-q} & =Y^{t+1}\left(x^{q} y^{-q}\right)^{-s} y^{-1}\left(z^{q} y^{-q}\right)^{-s} y^{q} z^{-q} \\
& =Y^{t+1}\left(x^{q} y^{-q}\right)^{-s-1} x^{q} y^{-q-1}\left(z^{q} y^{-q}\right)^{-s-1}<1 .
\end{aligned}
$$

Therefore $Z^{t} z^{q} y^{-q} Y^{-t}>1$. We have also that

$$
\begin{aligned}
X^{t} x^{q} y^{-q} & =X^{t+1}\left(z^{q} x^{-q}\right)^{-s} x^{-1}\left(y^{q} x^{-q}\right)^{-s-1} \\
& =X^{t+1}\left(z^{q} x^{-q}\right)^{-s} z^{q} x^{-q-1}\left(y^{q} x^{-q}\right)^{-s-1}>1
\end{aligned}
$$

Thus $X^{t} x^{q} y^{-q} Y^{-t}>1$. Therefore by $r_{2}^{\prime}$ we have a contradiction.

(3) The only subcase which is left to show is if $Y>1$ and $Z>1$. We have two subsubcases

- If $z^{q} y^{-q}>1$ then by subcase (2) $Y^{t} y^{q} x^{-q} X^{-t}=\left(X^{t} x^{q} y^{-q} Y^{-t}\right)^{-1}<1$ and by subcase (1) $Z^{t} z^{q} x^{-q} X^{-t}=\left(X^{t} x^{q} z^{-q} Z^{-t}\right)^{-1}<1$. Therefore we have a contradiction by $r_{1}^{\prime}$.

- If $z^{q} y^{-q}<1$ then by subcase (2) we have $Y^{t} y^{q} x^{-q} X^{-t}=\left(X^{t} x^{q} y^{-q} Y^{-t}\right)^{-1}<1$ and we have $Z^{t} z^{q} x^{-q} X^{-t}=Z^{t} z^{q} y^{-q} y q x^{-q} X^{-t}$ and by subcase (2) $y q x^{-q} X^{-t}<1$, so $Z^{t} z^{q} x^{-q} X^{-t}=Z^{t} z^{q} y^{-q} y q x^{-q} X^{-t}<1$. Therefore we have a contradiction by $r_{1}^{\prime}$.

Fith case: If $q<0, s>0, t>0$ and $l>0$.

We have $X=\left(y^{q} x^{-q}\right)^{s} x\left(z^{q} x^{-q}\right)^{s}>1$. Similarly as the first case we have three subcases:

(1) If $Y>1$ then since $Z Y X=1$ and $X>1$ then $Z<1$. Since $Y=\left(z^{q} y^{-q}\right)^{s} y\left(x^{q} y^{-q}\right)^{s}>1$, so $z^{q} y^{-q}>1$. we have,

$$
\begin{aligned}
X^{t} x^{q} z^{-q} & =X^{t-1}\left(y^{q} x^{-q}\right)^{s} x\left(z^{q} x^{-q}\right)^{s} x^{q} z^{-q} \\
& =X^{t-1}\left(y^{q} x^{-q}\right)^{s} x\left(z^{q} x^{-q}\right)^{s-1} z^{q} x^{-q} x^{q} z^{-q} \\
& =X^{t-1}\left(y^{q} x^{-q}\right)^{s} x\left(z^{q} x^{-q}\right)^{s-1}>1
\end{aligned}
$$

Therefore $X^{t} x^{q} z^{-q} Z^{-t}>1$ and $X^{t} x^{q} z^{-q} Z^{-t+1}>1$. We have also that,

$$
Z^{t} z^{q} y^{-q}=Z^{t-1}\left(x^{q} z^{-q}\right)^{s} z\left(y^{q} z^{-q}\right)^{s-1}<1
$$


Hence $y^{q} z^{-q} Z^{-t}>1$ and $Y^{t} y^{q} z^{-q} Z^{-t}>1$, so we have a contradiction by $r_{3}^{\prime \prime}$.

(2) If $Z>1$ then since $Z Y X=1$ and $X>1$ then $Y<1$. Since $Z=\left(x^{q} z^{-q}\right)^{s} z\left(y^{q} z^{-q}\right)^{s}$, $y^{q} z^{-q}>1$. We have,

$$
\begin{aligned}
Y^{t} y^{q} z^{-q} & =Y^{t-1}\left(z^{q} y^{-q}\right)^{s} y\left(x^{q} y^{-q}\right)^{s} y^{q} z^{-q} \\
& =Y^{t-1}\left(z^{q} y^{-q}\right)^{s} y\left(x^{q} y^{-q}\right)^{s-1} x^{q} y^{-q} y^{q} z^{-q} \\
& =Y^{t-1}\left(z^{q} y^{-q}\right)^{s} y\left(x^{q} y^{-q}\right)^{s-1} x^{q} z^{-q}<1
\end{aligned}
$$

Therefore $Y^{t} y^{q} z^{-q} Z^{-t}<1$, so $Z^{t} z^{q} y^{-q} Y^{-t}>1$ and $Z^{t} z^{q} y^{-q} Y^{-t+1}>1$ for $t>1$. For $t=1$, the relation $r_{2}^{\prime \prime}$ become

$$
r_{2}^{\prime \prime \prime}=\left(Z z^{q} y^{-q} Y^{-1}\right)^{l-1} Z z^{q} y^{-q} X x^{q} y^{-q} Y^{-1}\left(X x^{q} y^{-q} Y^{-1}\right)^{l-1}=1
$$

and we have

$$
z^{q} y^{-q} X=z^{q} y^{-q} y^{q} x^{-q}\left(y^{q} x^{-q}\right)^{s-1} x\left(z^{q} x^{-q}\right)^{s}=z^{q} x^{-q}\left(y^{q} x^{-q}\right)^{s-2} y^{q} x^{-q+1}\left(z^{q} x^{-q}\right)^{s}>1 .
$$

We have also that,

$$
\begin{aligned}
Y^{t} y^{q} x^{-q} & =Y^{t-1}\left(z^{q} y^{-q}\right)^{s} y\left(x^{q} y^{-q}\right)^{s} y^{q} x^{-q} \\
& =Y^{t-1}\left(z^{q} y^{-q}\right)^{s} y\left(x^{q} y^{-q}\right)^{s-1} x^{q} y^{-q} y^{q} x^{-q} \\
& =Y^{t-1}\left(z^{q} y^{-q}\right)^{s} y\left(x^{q} y^{-q}\right)^{s-1}<1
\end{aligned}
$$

Hence $Y^{t} y^{q} x^{-q} X^{-t}<1$ and $X^{t} x^{q} y^{-q} Y^{-t}>1$, so we have a contradiction by $r_{2}^{\prime \prime}$ and $r_{2}^{\prime \prime \prime}$.

(3) The only subcase which is left to show is when $Y<1$ and $Z<1$. We have two subsubcases

- If $z^{q} y^{-q}>1$ then by subcase (1) $Z^{t} z^{q} x^{-q} X^{-t}<1$. We have,

$$
\begin{aligned}
X^{t} x^{q} y^{-q} & =X^{t-1}\left(y^{q} x^{-q}\right)^{s} x\left(z^{q} x^{-q}\right)^{s} x^{q} y^{-q} \\
& =X^{t-1}\left(y^{q} x^{-q}\right)^{s} x\left(z^{q} x^{-q}\right)^{s-1} z^{q} x^{-q} x^{q} y^{-q} \\
& =X^{t-1}\left(y^{q} x^{-q}\right)^{s} x\left(z^{q} x^{-q}\right)^{s-1} z^{q} y^{-q}>1
\end{aligned}
$$

Therefore $X^{t} x^{q} y^{-q} Y^{-t}>1$, so $Y^{t} y^{q} x^{-q} X^{-t}<1$ and $Y^{t} y^{q} x^{-q} X^{-t+1}<1$ for $t>1$. Hence we have a contradiction by $r_{1}^{\prime \prime}$ if $t>1$. If $t=1$ use a similar argument as the previous case to conclude.

- If $z^{q} y^{-q}<1$ then $y^{q} z^{-q}>1$. We have $Y^{t} y^{q} x^{-q} X^{-t}<1$ and $Y^{t} y^{q} x^{-q} X^{-t+1}<1$ by subcase (2). We also have $Z^{t} z^{q} x^{-q} X^{-t}<1$ by subcase (1). Therefore we have a contradiction by $r_{1}^{\prime}$.

Sixth case: $q<0, s>0, t<0$ and $l<0$.

We have $X=\left(y^{q} x^{-q}\right)^{s} x\left(z^{q} x^{-q}\right)^{s}>1$. Similarly as the first case we have three subcases:

(1) If $Y>1$ then since $Z Y X=1$ then $Z<1$. Since $Y=\left(z^{q} y^{-q}\right)^{s} y\left(x^{q} y^{-q}\right)^{s}>1, z^{q} y^{-q}>1$. The relation $r_{3}^{\prime}$ become $r_{3}^{\prime \prime \prime}=\left(X^{t} x^{q} z^{-q} Z^{-t}\right)^{l} Z^{t+1} z^{q} y^{-q} Y^{-t}\left(Y^{t} y^{q} z^{-q} Z^{-t}\right)^{l+1}=1$ and this implies a contradiction. 
(2) If $Z>1$ then since $Z Y X=1$ then $Y<1$. Since $Z=\left(x^{q} z^{-q}\right)^{s} z\left(y^{q} z^{-q}\right)^{s}, y^{q} z^{-q}>1$. The relation $r_{2}^{\prime}$ become $r_{2}^{\prime \prime \prime}=\left(Z^{t} z^{q} y^{-q} Y^{-t}\right)^{l} Y^{t+1} y^{q} x^{-q} X^{-t}\left(X^{t} x^{q} y^{-q} Y^{-t}\right)^{l+1}=1$, which implies a contradiction.

(3) The only subcase which is left to show is when $Y<1$ and $Z<1$ and in this subcase we have a contradiction by the following: $r_{1}^{\prime \prime \prime}=\left(Y^{t} y^{q} x^{-q} X^{-t}\right)^{l} X^{t+1} x^{q} z^{-q} Z^{-t}\left(Z^{t} z^{q} x^{-q} X^{-t}\right)^{l+1}=$ 1 .

Seventh case: $q>0, s<0, t<0$ and $l>0$.

We have $X=\left(y^{q} x^{-q}\right)^{s} x\left(z^{q} x^{-q}\right)^{s}>1$. Similarly as the first case we have three subcases:

(1) If $Y>1$ then since $Z Y X=1$ then $Z<1$. Since $Y=\left(z^{q} y^{-q}\right)^{s} y\left(x^{q} y^{-q}\right)^{s}>1, z^{q} y^{-q}<1$. We have

$$
\begin{aligned}
x^{q} z^{-q} Z^{-t} & =x^{q} z^{-q}\left(x^{q} z^{-q}\right)^{s} z\left(y^{q} z^{-q}\right)^{s} Z^{-t-1} \\
& =x^{q} z^{-q}\left(z^{q} x^{-q}\right)^{-s} z\left(y^{q} z^{-q}\right)^{s} Z^{-t-1} \\
& =x^{q} z^{-q} z^{q} x^{-q}\left(z^{q} x^{-q}\right)^{-s-1} z\left(y^{q} z^{-q}\right)^{s} Z^{-t-1} \\
& =\left(z^{q} x^{-q}\right)^{-s-1} z\left(y^{q} z^{-q}\right)^{s} Z^{-t-1}<1
\end{aligned}
$$

Therefore $X^{t} x^{q} z^{-q} Z^{-t}<1$. We have also that,

$$
\begin{aligned}
y^{q} z^{-q} Z^{-t} & =y^{q} z^{-q}\left(x^{q} z^{-q}\right)^{s} z\left(y^{q} z^{-q}\right)^{s} Z^{-t-1} \\
& =y^{q} z^{-q}\left(z^{q} x^{-q}\right)^{-s} z\left(y^{q} z^{-q}\right)^{s} Z^{-t-1} \\
& =y^{q} z^{-q} z^{q} x^{-q}\left(z^{q} x^{-q}\right)^{-s-1} z\left(y^{q} z^{-q}\right)^{s} Z^{-t-1} \\
& =y^{q} x^{-q}\left(z^{q} x^{-q}\right)^{-s-1} z\left(y^{q} z^{-q}\right)^{s} Z^{-t-1}<1
\end{aligned}
$$

Therefore $Y^{t} y^{q} z^{-q} Z^{-t}<1$. Thus we have a contradiction by $r_{3}^{\prime}$.

(2) If $Z>1$ then since $Z Y X=1$ then $Y<1$. Since $Z=\left(x^{q} z^{-q}\right)^{s} z\left(y^{q} z^{-q}\right)^{s}, y^{q} z^{-q}<1$.

We have,

$$
\begin{aligned}
z^{q} y^{-q} Y^{-t} & =z^{q} y^{-q}\left(z^{q} y^{-q}\right)^{s} y\left(x^{q} y^{-q}\right)^{s} Y^{-t-1} \\
& =\left(z^{q} y^{-q}\right)^{s+1} y\left(x^{q} y^{-q}\right)^{s-1} Y^{-t-1}<1
\end{aligned}
$$

Hence $Z^{t} z^{q} y^{-q} Y^{-t}<1$. We also have,

$$
\begin{aligned}
X^{t} x^{q} y^{-q} & =X^{t+1}\left(z^{q} x^{-q}\right)^{-s} x^{-1}\left(y^{q} x^{-q}\right)^{-s} x^{q} y^{-q} \\
& =X^{t+1}\left(z^{q} x^{-q}\right)^{-s} x^{-1}\left(y^{q} x^{-q}\right)^{-s-1}<1
\end{aligned}
$$

Therefore $X^{t} x^{q} y^{-q} Y^{-t}<1$, and we have a contradiction by $r_{2}^{\prime}$.

(3) The only subcase which is left to show is when $Y<1$ and $Z<1$ and in this subcase we have two subsubcases:

- If $z^{q} y^{-q}>1$ then by subcase (2) we have $X^{t} x^{q} y^{-q}<1$, so $y^{q} x^{-q} X^{-t}>1$. Therefore we have a contradiction by the following:

$$
r_{1}^{\prime}=\left(Y^{t} y^{q} x^{-q} X^{-t}\right)^{l} X\left(Z^{t} z^{q} y^{-q} y^{q} x^{-q} X^{-t}\right)^{l}=1
$$

- If $z^{q} y^{-q}<1$ then by subcase (1) $X^{t} x^{q} z^{-q} Z^{-t}<1$, so $Z^{t} z^{q} x^{-q} X^{-t}>1$. We have also by subcase (2) $X^{t} x^{q} y^{-q} Y^{-t}<1$, so $Y^{t} y^{q} x^{-q} X^{-t}>1$, and we have a contradiction by $r_{1}^{\prime}$. 
Eighth case: $q>0, s>0, t<0$ and $l<0$.

We have $X=\left(y^{q} x^{-q}\right)^{s} x\left(z^{q} x^{-q}\right)^{s}=\left(y^{q} x^{-q}\right)^{s-1} y^{q} x^{-q+1}\left(z^{q} x^{-q}\right)^{s}<1$.

We discuss the signs of $Y$ and $Z$. We have three subcases

(1) If $Y<1$, then since $Z Y X=1$ and $X<1$ then $Z>1$. Since $Y=\left(z^{q} y^{-q}\right)^{s} y\left(x^{q} y^{-q}\right)^{s}=$ $\left(z^{q} y^{-q}\right)^{s} y x x^{q-1} y^{-q}\left(x^{q} y^{-q}\right)^{s-1}=\left(z^{q} y^{-q}\right)^{s} z^{-1} x^{q-1} y^{-q}\left(x^{q} y^{-q}\right)^{s-1}$ then $z^{q} y^{-q}<1$, and $y^{q} z^{-q}>1$. We have a contradiction by the following:

$$
r_{3}^{\prime}=\left(X^{t} x^{q} z^{-q} Z^{-t}\right)^{l} Z^{t+1} z^{q} y^{-q} Y^{-t}\left(Y^{t} y^{q} z^{-q} Z^{-t}\right)^{l+1}=1
$$

(2) If $Z<1$ then since $Z Y X=1$ and $X<1$ then $Y>1$. Since $Z=\left(x^{q} z^{-q}\right)^{s} z\left(y^{q} z^{-q}\right)^{s}=$ $\left(x^{q} z^{-q}\right)^{s-1} x^{q} z^{-q+1}\left(y^{q} z^{-q}\right)^{s}$ then $y^{q} z^{-q}<1$, so $z^{q} y^{-q}>1$. We have a contradiction by the following:

$$
r_{2}^{\prime}=\left(Z^{t} z^{q} y^{-q} Y^{-t}\right)^{l} Y^{t+1} y^{q} x^{-q} X^{-t}\left(X^{t} x^{q} y^{-q} Y^{-t}\right)^{l+1}=1
$$

(3) The only subcase which is left to check is if $Y>1$ and $Z>1$. In this subcase we have a contradiction by the following:

$$
r_{1}^{\prime}=\left(Y^{t} y^{q} x^{-q} X^{-t}\right)^{l} X^{t+1} x^{q} z^{-q} Z^{-t}\left(Z^{t} z^{q} x^{-q} X^{-t}\right)^{l+1}=1
$$

This complete the proof of Theorem 4.1.

Remark 4.2. (1) Since the knot $5_{1}$ corresponds to the two-bridge knot $K_{[-2,2,-2,2]}$ and, by Gordon and Lidman [GL], the fundamental group of the $n$-fold cyclic branched cover of $5_{1}$ is left-orderable for $n \geq 4$, then another question we can also ask is the following: Is the fundamental group of the $\mathrm{n}$-fold cyclic branched cover of $K_{[-2 q, 2 s,-2 t, 2 l]}$ left-orderable for $n \geq 4$ ?

(2) In general, it is not true that for every 2-bridge knot $K$ the fundamental group of the 3fold cyclic branched cover of $K$ is not left-orderable, as an example we have the 2-bridge knot $K_{[6,-3]}$ which can also be written as the genus three 2-bridge knot $K_{[-2,2,-2,2,-2,-2]}$. By [GL] this knot has left-orderable fundamental group. The proof come from the fact that $\pi_{1}\left(\Sigma_{3}\left(K_{[6,-3]}\right)\right)$ has a nontrivial representation into $P S L(2, \mathbb{R})$. Since $\Sigma_{3}\left(K_{[6,-3]}\right)$ is an integer homology sphere, this representation lifts to a nontrivial representation into $\widetilde{S L}(2, \mathbb{R})$. Since $\widetilde{S L}(2, \mathbb{R})$ is left-orderable then $\pi_{1}\left(\Sigma_{3}\left(K_{[6,-3]}\right)\right)$ is also left-orderable.

\section{L-SPACES AND GENUS 2 TWO-BRIDGE KNOTS}

In this section we will complete the proof of Theorem 1.2 by proving the following theorem.

Theorem 5.1. The 3-fold cyclic branched cover of $K_{[-2 q, 2 s,-2 t, 2 l]}$ is an L-space, where $q, s, t$ and $l \in \mathbb{Z} \backslash\{0\}$. 
In order to show that the 3 -fold cyclic branched cover of any genus 2 two-bridge knot $K_{[-2 q, 2 s,-2 t, 2 l]}$ is an L-space we have to consider all cases for the signs of $q, s, t$ and $l$. We have sixteen such cases, but since the mirror image of $K_{[-2 q, 2 s,-2 t, 2 l]}$ is $K_{[2 q,-2 s, 2 t,-2 l]}$ we need only deal with eight of them:

(1) $q>0, s>0, t>0$ and $l>0$;

(2) $q<0, s>0, t<0$ and $l>0$;

(3) $q>0, s<0, t>0$ and $l>0$;

(4) $q<0, s<0, t<0$ and $l>0$;

(5) $q>0, s>0, t<0$ and $l>0$

(6) $q>0, s<0, t<0$ and $l<0$;

(7) $q>0, s<0, t<0$ and $l>0$

(8) $q<0, s<0, t>0$ and $l>0$.

By $A(t ; *, *, *)$ we mean the link $A$ pictured in Figure 3. Let $\gamma \in\{\infty, 0\}$. By $A(t ; \gamma, *, *)$ we mean the link $A$ with the resolution $\gamma$ (cf. Figure 2) at the leftmost $t$ half twists (see Figures 5 and 6 for the case $t>1)$. By $A(t ; *, \gamma, *)$ we mean the link $A$ with the resolution $\gamma$ at the $t$ half twists on the middle (see Figures 7, 8, 9 and 10 for the case $t>1$ ) and by $A(t ; *, *, \gamma$ ) we mean the link $A$ with the resolution $\gamma$ at the $t$ half twists on the right (see Figures 11, 12, 13, 14, 15 and 16 for the case $t>1$ ). Similar notation will be used also for $L(l ; *, *, *)$ (cf. Figure 17).

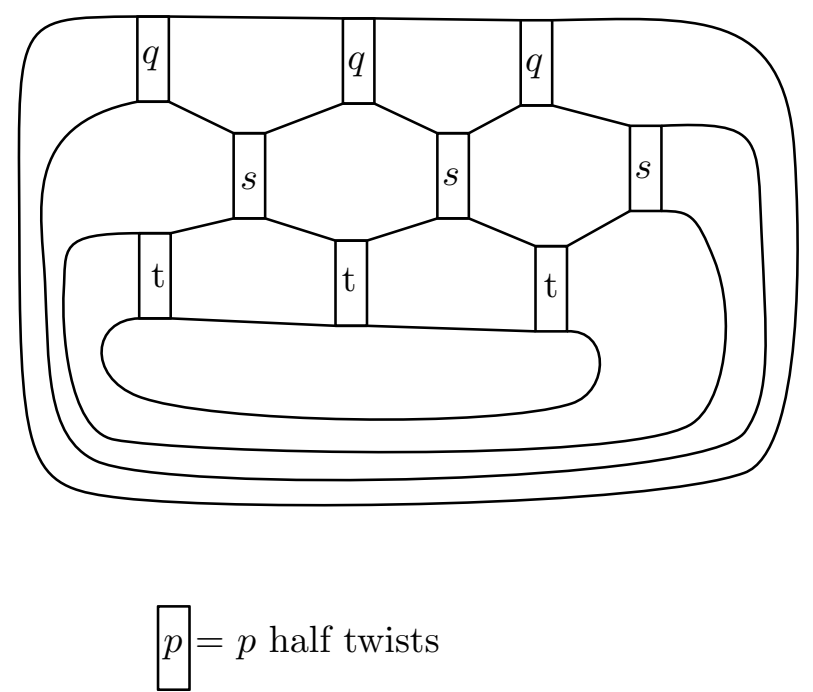

Figure 3. The link $A$. 


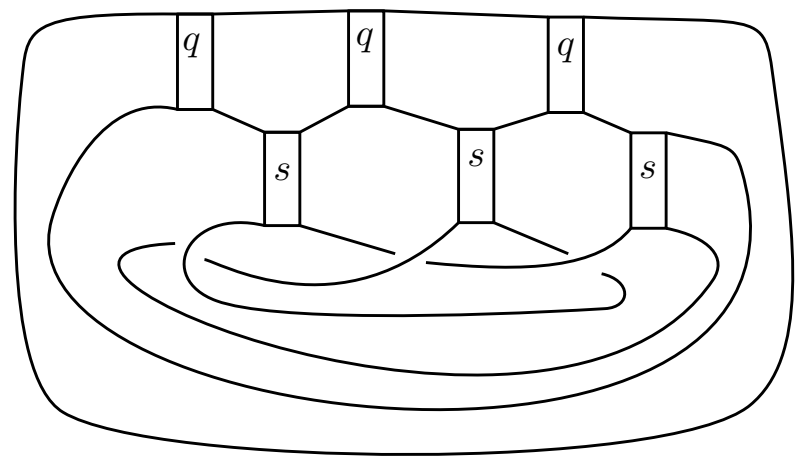

Figure 4 . The link $A$ for $t=1$

The proof of Theorem 5.1 will be split into two parts. First, we will study the link $A(t ; *, *, *)$. That is, we study when it is quasi-alternating or when $\Sigma_{2}(A(t ; *, *, *))$ is an $L$-space. Second, we use the link $A(t ; *, *, *)$ to show that $\Sigma_{2}(L(l ; *, *, *))$ is an $L$-space.

5.1. The link $A(t ; *, *, *)$. We have 4 cases,

1) If $q>0, s>0, t>0$

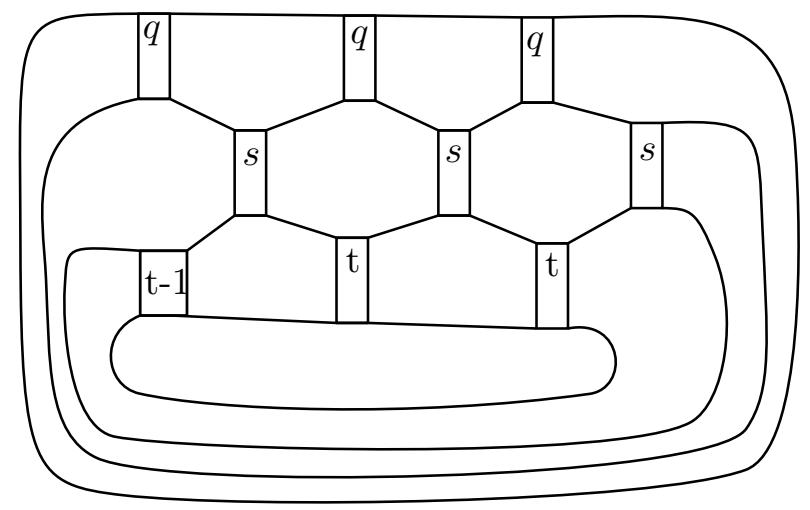

Figure 5. The link $A(t ; \infty, *, *)$.

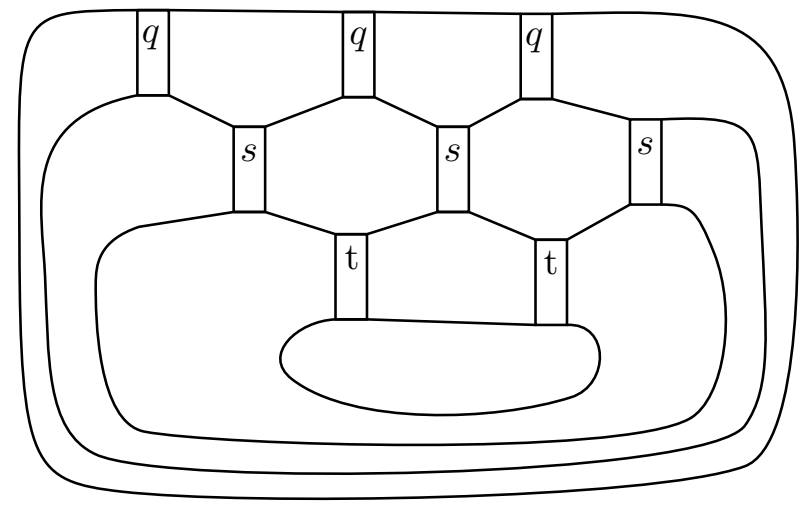

Figure 6 . The link $A(t ; 0, *, *)$. 


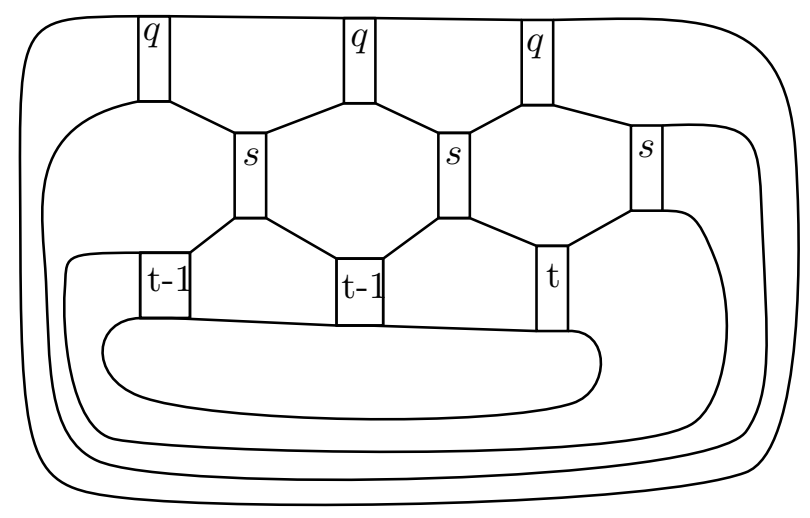

Figure 7 . The link $A(t ; \infty, \infty, *)$.

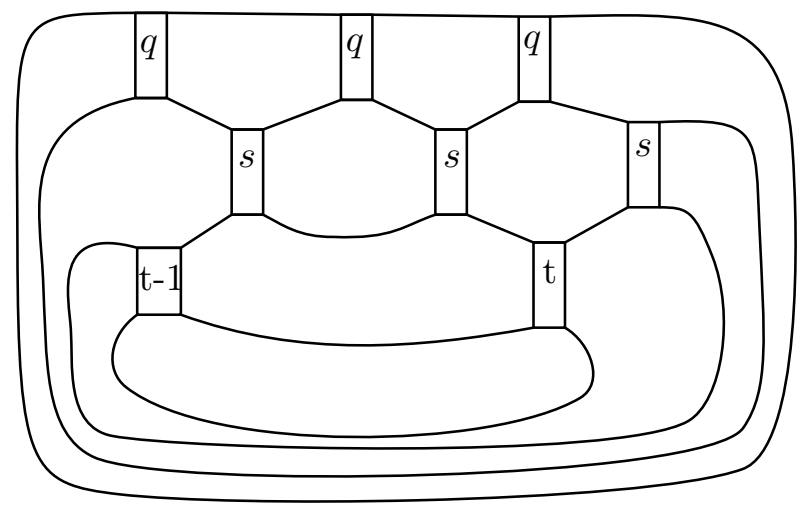

Figure 8 . The link $A(t ; \infty, 0, *)$.

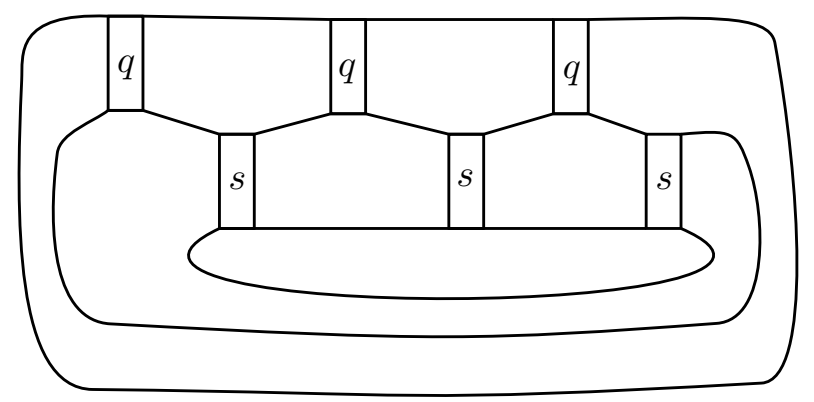

Figure 9. The link $A(t ; 0,0, *)$. 


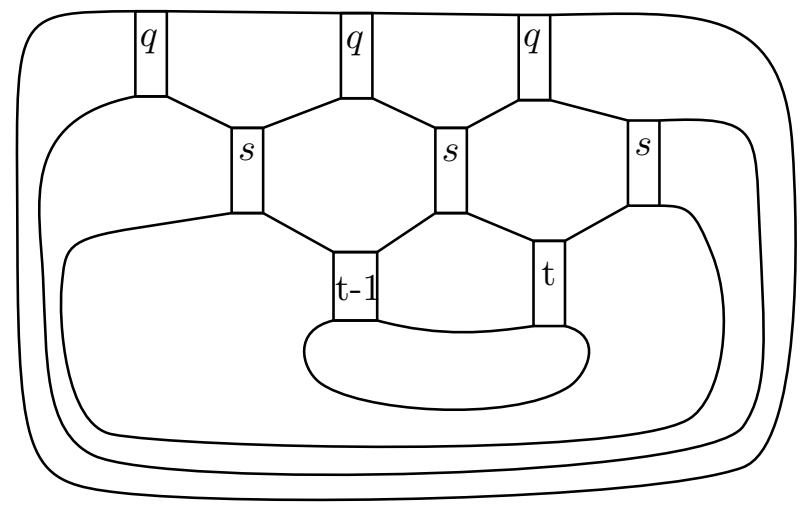

Figure 10. The link $A(t ; 0, \infty, *)$.

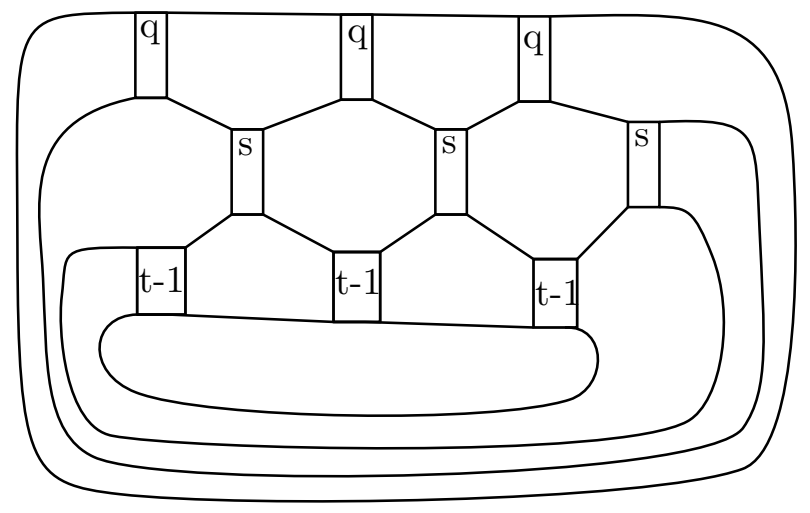

Figure 11. The link $A(t ; \infty, \infty, \infty)$.

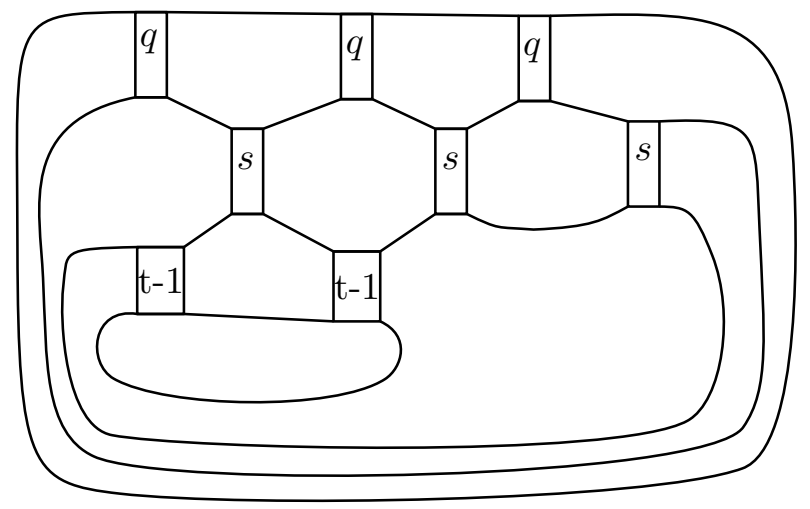

Figure 12. The link $A(t ; \infty, \infty, 0)$. 


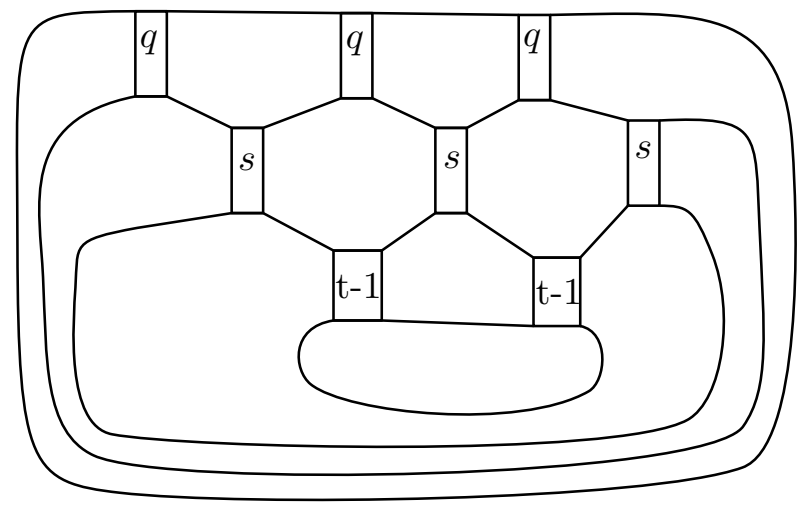

Figure 13. The link $A(t ; 0, \infty, \infty)$.

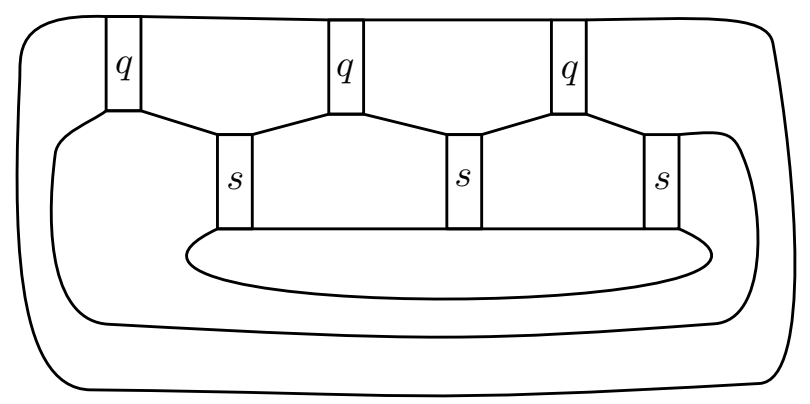

Figure 14. The link $A(t ; 0, \infty, 0)$.

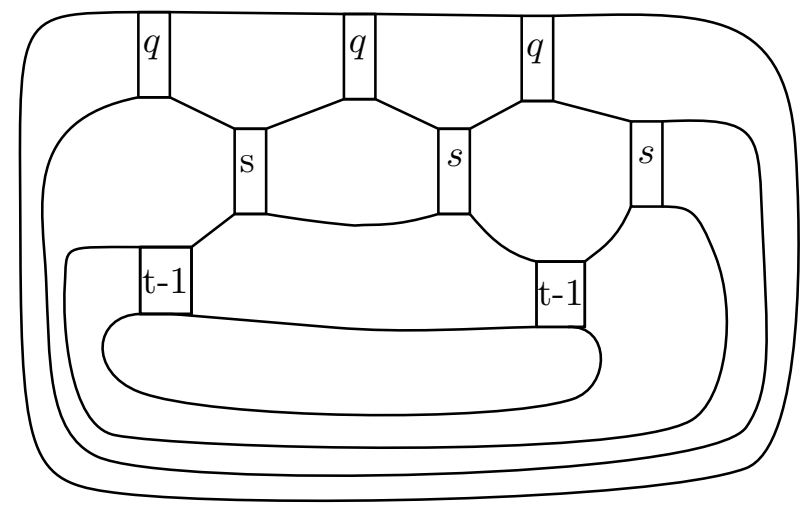

Figure 15 . The link $A(t ; \infty, 0, \infty)$. 


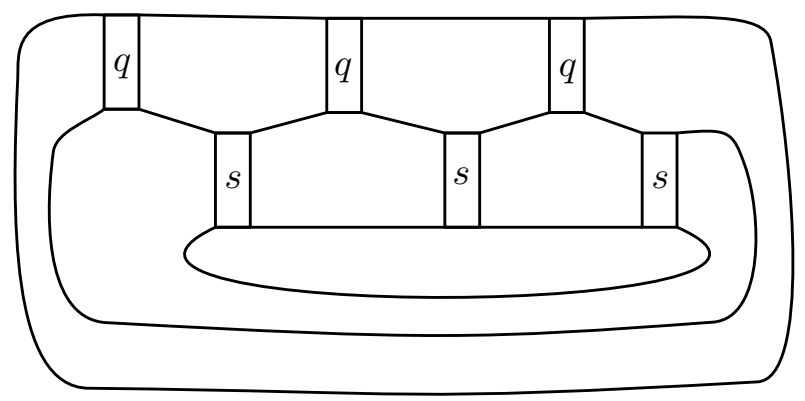

Figure 16. The link $A(t ; \infty, 0,0)$.

The Goeritz matrix of $A(t ; *, *, *)$ is

$$
\left[\begin{array}{ccccccccc}
-2 I & I & O & \ldots & O & \ldots & & & \\
I & -2 I & I & O & \ldots & O & \ldots & & \\
\vdots & \vdots & \vdots & \ddots & \vdots & \vdots & \vdots & & \\
O & O \ldots & O & I & S & I & O \ldots & & \\
O & \ldots & O & O & I & -2 I & I & O \ldots & \\
\vdots & \vdots & \vdots & & \vdots & \ddots & \vdots & \vdots & \vdots \\
O & \ldots & O & O & \ldots O & I & -2 I & I & F \\
O & O & O & \ldots & \ldots & O & I & -2 I & E \\
Z & Z & \ldots & \ldots & Z & Z & Z & D & -3
\end{array}\right]
$$

where

$$
\begin{gathered}
S=\left[\begin{array}{ccc}
2 s-2 & -s & -s \\
-s & 2 s-2 & -s \\
-s & -s & 2 s-2
\end{array}\right], F=\left[\begin{array}{l}
0 \\
0 \\
0
\end{array}\right] \\
E=\left[\begin{array}{l}
1 \\
1 \\
1
\end{array}\right] \\
O=\left[\begin{array}{lll}
0 & 0 & 0 \\
0 & 0 & 0 \\
0 & 0 & 0
\end{array}\right], I=\left[\begin{array}{lll}
1 & 0 & 0 \\
0 & 1 & 0 \\
0 & 0 & 1
\end{array}\right] \\
Z=\left[\begin{array}{lll}
0 & 0 & 0
\end{array}\right], D=\left[\begin{array}{lll}
1 & 1 & 1
\end{array}\right]
\end{gathered}
$$

a) By induction, assume $t=1$.

In the following table we give the determinant of the corresponding link, obtained through brute calculation. 


\begin{tabular}{|l|l|}
\hline Link & Determinant $(s>1)$ \\
\hline$A(t=1 ; *, *, *)$ & $3(-1-q+3 q s)^{2}$ \\
\hline$A(t=1 ; 0, *, *)$ & $2(-1-q+3 q s)(-1+3 q s)$ \\
\hline$A(t=1 ; \infty, *, *)$ & $(-1-q+3 q s)(-1-3 q+3 q s)$ \\
\hline$A(t=1 ; 0, \infty, *)$ & $(-1+3 q s)(-1-2 q+3 q s)$ \\
\hline$A(t=1 ; 0,0, *)$ & $(-1+3 q s)^{2}$ \\
\hline
\end{tabular}

TABLE 2

Lemma 5.2. The links $A(t=1 ; \infty, *, *), A(t=1 ; 0, \infty, *)$ and $A(t=1 ; 0,0, *)$ are quasialternating for $s>1$. Therefore, $A(t=1 ; 0, *, *)$ and $A(t=1 ; *, *, *)$ are also quasi-alternating for $s>1$.

Proof. The fact that the links $A(t=1 ; \infty, *, *), A(t=1 ; 0, \infty, *)$ and $A(t=1 ; 0,0, *)$ are quasialternating for $s>1$ was shown by Peters [P]. Since $\operatorname{det} A(t=1 ; 0, *, *)=\operatorname{det} A(t=1 ; 0, \infty, *)$ $+\operatorname{det} A(t=1 ; 0,0, *)$ and $\operatorname{det} A(t=1 ; *, *, *)=\operatorname{det} A(t=1 ; 0, *, *)+\operatorname{det} A(t=1 ; \infty, *, *)$ for $s>1$ by Table 2 , then $A(t=1 ; 0, *, *)$ and $A(t=1 ; *, *, *)$ are quasi-alternating for $s>1$.

b) Assume $t>1$.

We have the following table which gives the determinant of the corresponding link.

\begin{tabular}{|l|l|}
\hline Link & Determinant $(t>1)$ \\
\hline$A(t ; *, *, *)$ & $3(-t-q+3 q s t)^{2}$ \\
\hline$A(t ; 0, *, *)$ & $2(-t-q+3 q s t)(-1+3 q s)$ \\
\hline$A(t ; \infty, *, *)$ & $(-t-q+3 q s t)(2-3 q-6 q s-3 t+9 q s t)$ \\
\hline$A(t ; 0, \infty, *)$ & $(-1+3 q s)(1-2 q-3 q s-2 t+6 q s t)$ \\
\hline$A(t ; \infty, 0, *)$ & $(-1+3 q s)(1-2 q-3 q s-2 t+6 q s t)$ \\
\hline$A(t ; 0,0, *)$ & $(-1+3 q s)^{2}$ \\
\hline$A(t ; \infty, \infty, *)$ & $(1-q-3 q s-t+3 q s t)(1-3 q-3 q s-3 t+9 q s t)$ \\
\hline$A(t ; \infty, \infty, \infty)$ & $3(1-q-3 q s-t+3 q s t)^{2}$ \\
\hline$A(t ; 0, \infty, \infty)$ & $2(-1+3 q s)(1-q-3 q s-t+3 q s t)$ \\
\hline
\end{tabular}

TABLE 3

Lemma 5.3. (1) $A(t ; 0,0, *)=A(t ; \infty, 0,0)=A(t ; 0, \infty, 0)$,

(2) $A(t ; \infty, 0, \infty)=A(t ; \infty, \infty, 0)=A(t ; 0, \infty, \infty)$,

(3) $A(t ; \infty, \infty, \infty)=A(t-1 ; *, *, *)$,

(4) $A(t ; 0, \infty, \infty)=A(t-1 ; 0, *, *)$,

(5) $A(t ; 0,0, *)=A(t=1 ; 0,0, *)$.

(6) $A(q=s=t=1 ; *, *, *)=T(3,4)$ and $A(q=s=t=1 ; 0, *, *)=P(2,-3,-2)$.

Proof. (1) Figure 9, Figure 14 and Figure 16 are the same. 
(2) By applying ambient isotopy, one can see that Figure 12, Figure 13 and Figure 15 depict the same links.

(3) Replacing $t$ by $t-1$ in Figure 3 and considering Figure 11, one can see that $A(t ; \infty, \infty, \infty)$ $=A(t-1 ; *, *, *)$.

(4) Replacing $t$ by $t-1$ in Figure 6 and considering Figure 13, one can see that $A(t ; 0, \infty, \infty)$ $=A(t-1 ; 0, *, *)$.

(5) Figure 9 does not depends on $t$.

(6) This comes from direct computation of the links.

Lemma 5.4. (1) $\operatorname{det} A(t ; *, *, *)=\operatorname{det} A(t ; 0, *, *)+\operatorname{det} A(t ; \infty, *, *)$

(2) $\operatorname{det} A(t ; 0, *, *)=\operatorname{det} A(t ; 0, \infty, *)+\operatorname{det} A(t ; 0,0, *)$

(3) $\operatorname{det} A(t ; \infty, *, *)=\operatorname{det} A(t ; \infty, 0, *)+\operatorname{det} A(t ; \infty, \infty, *)$

(4) $\operatorname{det} A(t ; 0, \infty, *)=\operatorname{det} A(t ; 0, \infty, 0)+\operatorname{det} A(t ; 0, \infty, \infty)$

(5) $\operatorname{det} A(t ; \infty, 0, *)=\operatorname{det} A(t ; 0, \infty, 0)+\operatorname{det} A(t ; 0, \infty, \infty)$

(6) $\operatorname{det} A(t ; \infty, \infty, *)=\operatorname{det} A(t ; 0, \infty, \infty)+\operatorname{det} A(t ; \infty, \infty, \infty)$

Proof. Table 3.

Claim 5.5. The link $A(t ; *, *, *)$ is quasi-alternating for $s>1$.

Proof. We have shown that the link $A(t=1 ; *, *, *)$ is quasi-alternating for $s>1$. By induction assume $t>1$ and that the link $A(t-1 ; *, *, *)$ is quasi-alternating. We will show that $A(t ; *, *, *)$ is quasi-alternating.

Since $A(t ; \infty, \infty, \infty)=A(t-1 ; *, *, *)$ and $A(t ; 0, \infty, \infty)=A(t-1 ; 0, *, *)$ are quasi-alternating by induction hypothesis, then $A(t ; \infty, \infty, *)$ is also quasi-alternating by Lemma 5.4. Since $A(t ; 0,0, *)=A(t=1 ;, 0,0, *)$ and $A(t=1 ;, 0,0, *)$ is quasi-alternating then also $A(t ; 0,0, *)$, and since $A(t ; 0,0, *)=A(t ; \infty, 0,0)=A(t ; 0, \infty, 0)$ and $A(t ; \infty, 0, \infty)=A(t ; \infty, \infty, 0)=$ $A(t ; 0, \infty, \infty)$ by Lemma 5.3 , then $A(t ; 0, \infty, *)$ and $A(t ; \infty, 0, *)$ are quasi-alternating by Lemma 5.4. Therefore, $A(t ; 0, *, *)$ and $A(t ; \infty, *, *)$ are also quasi-alternating by Lemma 5.4. Finaly the link $A(t ; *, *, *)$ is quasi-alternating by Lemma 5.4 .

This completes the proof that $A(t ; *, *, *)$ is quasi-alternating for $s>1$.

Claim 5.6. $\Sigma_{2}(A(t ; *, *, *))$ is an L-space for $s=1$. In particular $\Sigma_{2}(A(t ; \infty, \infty, *))$ is an $L$-space for $s=1$ and $t>1$.

Proof. We have two cases

(1) Assume first that $t=q$. We have that if $q=s=t=1$ then $A(*, *, *)=T(3,4)$ and $A(0, *, *)=P(2,-3,-2)$ by Lemma 5.3 , and $\Sigma_{2}(T(3,4))$ and $\Sigma_{2}(P(2,-3,-2))$ are Lspaces. By induction on $t$, assume $t>1$ and $\Sigma_{2}(A(t-1 ; *, *, *))$ and $\Sigma_{2}(A(t-1 ; 0, *, *))$ are L-spaces. We will show that $\Sigma_{2}(A(t ; 0, *, *))$ and $\Sigma_{2}(A(t ; *, *, *))$ are L-spaces. 
Since $A(t ; \infty, \infty, \infty)=A(t-1 ; *, *, *)$ and $A(t ; 0, \infty, \infty)=A(t-1 ; 0, *, *)$ then $\Sigma_{2}(A(t ; \infty, \infty, \infty))$ and $\Sigma_{2}(A(t ; 0, \infty, \infty))$ are L-spaces by induction hypothesis. Therefore, $\Sigma_{2}(A(t ; \infty, \infty, *))$ is an L-space by Lemma 5.4 ([OSz] $)$. Since $A(t ; 0,0, *)=A(t=$ $1 ;, 0,0, *)$ and $\Sigma_{2}(A(t=1 ;, 0,0, *))$ is an L-space, then $\Sigma_{2}(A(t ; 0,0, *))$ is also an Lspace. Since $A(t ; 0,0, *)=A(t ; \infty, 0,0)=A(t ; 0, \infty, 0)$ and $A(t ; \infty, 0, \infty)=A(t ; \infty, \infty, 0)$ $=A(t ; 0, \infty, \infty)$ by Lemma 5.3 , then $\Sigma_{2}(A(t ; 0, \infty, *))$ and $\Sigma_{2}(A(t ; \infty, 0, *))$ are L-space by Lemma 5.4. Therefore, $\Sigma_{2}\left((A(t ; 0, *, *))\right.$ and $\Sigma_{2}(A(t ; \infty, *, *))$ are also L-spaces. Finaly $\Sigma_{2}(A(t ; *, *, *))$ is an L-space by Lemma 5.4 for $t=q$.

(2) Fix $q$ and assume $t \geq q$. In case (1), we have shown that if $t=q$, then $\Sigma_{2}((A(t ; 0, *, *))$ and $\Sigma_{2}(A(t ; *, *, *))$ are also L-spaces. By induction on $t$, assume $t>q$ and $\Sigma_{2}(A(t-$ $1 ; *, *, *))$ and $\Sigma_{2}(A(t-1 ; 0, *, *))$ are L-spaces. We will show that $\Sigma_{2}(A(t ; 0, *, *))$ and $\Sigma_{2}(A(t ; *, *, *))$ are L-spaces.

Since $A(t ; \infty, \infty, \infty)=A(t-1 ; *, *, *)$ and $A(t ; 0, \infty, \infty)=A(t-1 ; 0, *, *)$ then $\Sigma_{2}(A(t ; \infty, \infty, \infty))$ and $\Sigma_{2}(A(t ; 0, \infty, \infty))$ are L-spaces by induction hypothesis. Therefore, $\Sigma_{2}(A(t ; \infty, \infty, *))$ is an L-space by Lemma 5.4 ([OSz]). Since $A(t ; 0,0, *)=A(t=$ $1 ;, 0,0, *)$ and $\Sigma_{2}(A(t=1 ;, 0,0, *))$ is an L-space, then $\Sigma_{2}(A(t ; 0,0, *))$ is also an Lspace. Since $A(t ; 0,0, *)=A(t ; \infty, 0,0)=A(t ; 0, \infty, 0)$ and $A(t ; \infty, 0, \infty)=A(t ; \infty, \infty, 0)$ $=A(t ; 0, \infty, \infty)$ by Lemma 5.3 , then $\Sigma_{2}(A(t ; 0, \infty, *))$ and $\Sigma_{2}(A(t ; \infty, 0, *))$ are L-space by Lemma 5.4. Therefore, $\Sigma_{2}\left((A(t ; 0, *, *))\right.$ and $\Sigma_{2}(A(t ; \infty, *, *))$ are also L-spaces by Lemma 5.4. Finaly $\Sigma_{2}(A(t ; *, *, *))$ is an L-space by Lemma 5.4 for $t \geq q$.

Since $q$ was arbitrary, then this is true for any $t$ and $q$ such that $t \geq q$. Since $t$ and $q$ are symmetric for $A$, then $\Sigma_{2}(A(t ; *, *, *))$ is an L-space for $s=1$.

2) If $q>0, s<0, t>0$ or $q<0, s>0, t<0$ then the link $A(t ; *, *, *)$ is alternating and therefore quasi-alternating.

3) If $q<0, s>0, t>0$ a similar argument as that of case 1$)$ shows when $A(t ; *, *, *)$ is quasi-alternating or when $\Sigma_{2}(A(t ; *, *, *))$ is an L-space.

4) If $q<0, s<0, t<0$, then the link $A(t ; *, *, *)$ is the mirror image of the link $A(t ; *, *, *)$ when $q>0, s>0, t>0$, so a similar argument as that of case 1) shows when it is quasi-alternating or when $\Sigma_{2}(A(t ; *, *, *))$ is an L-space.

5.2. Proof of Theorem 5.1. The following theorem is essential in the proof of Theorem 5.1.

Theorem 5.7. (Theorem 3 in $[\mathrm{MV}]$ ) The 3-fold cyclic branched cover of $K_{[-2 q, 2 s,-2 t, 2 l]}$ is the 2-fold branched cover of the link $L(l ; *, *, *)$.

To prove Theorem 5.1 we have eight cases:

(1) If $q>0, s>0, t>0, l>0$. 


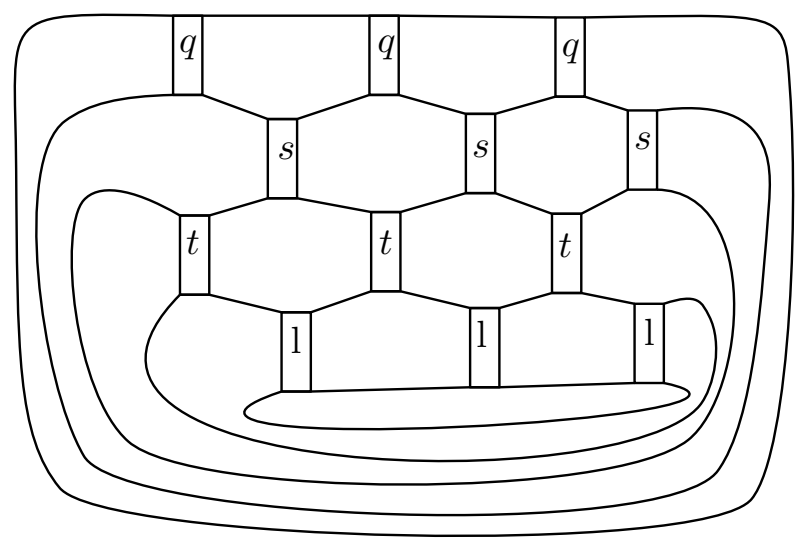

Figure 17. The link $L$.

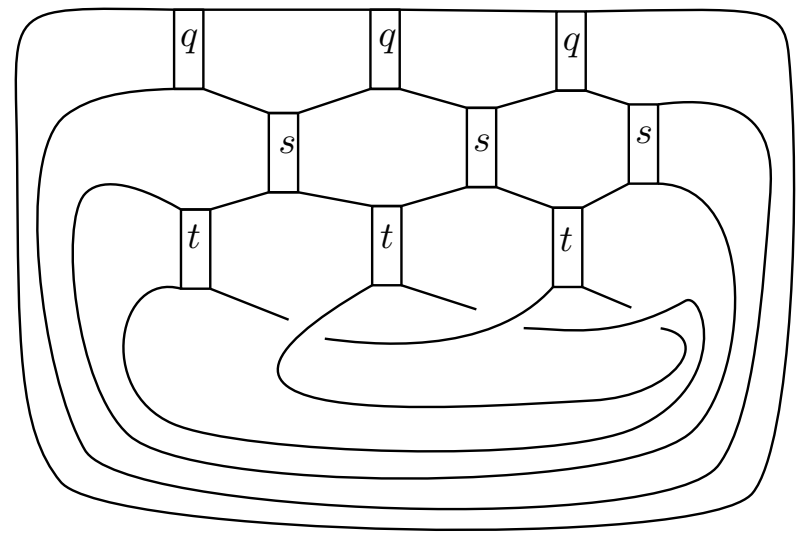

Figure 18 . The link $L$ for $l=1$.

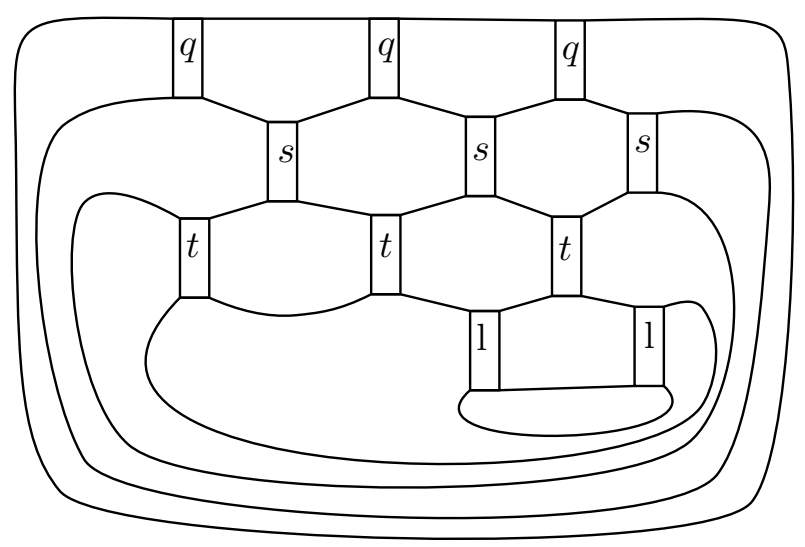

Figure 19 . The link $L(l ; 0, *, *)$. 


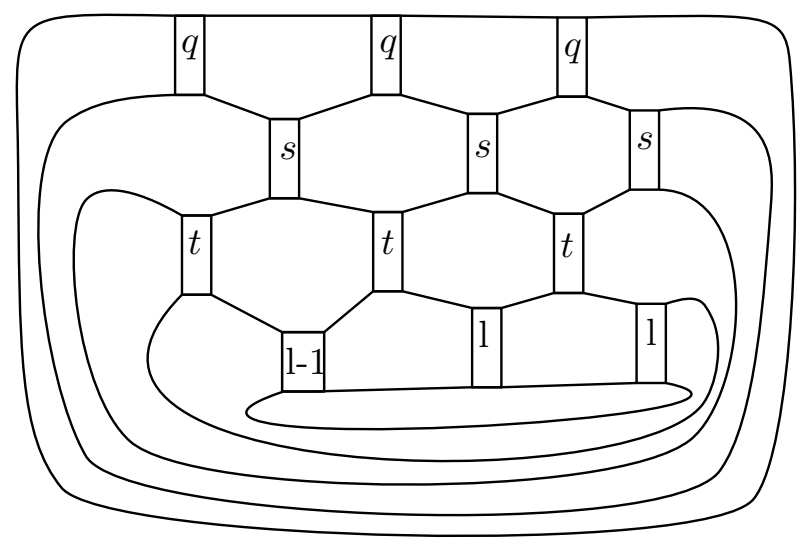

Figure 20. The link $L(l ; \infty, *, *)$.

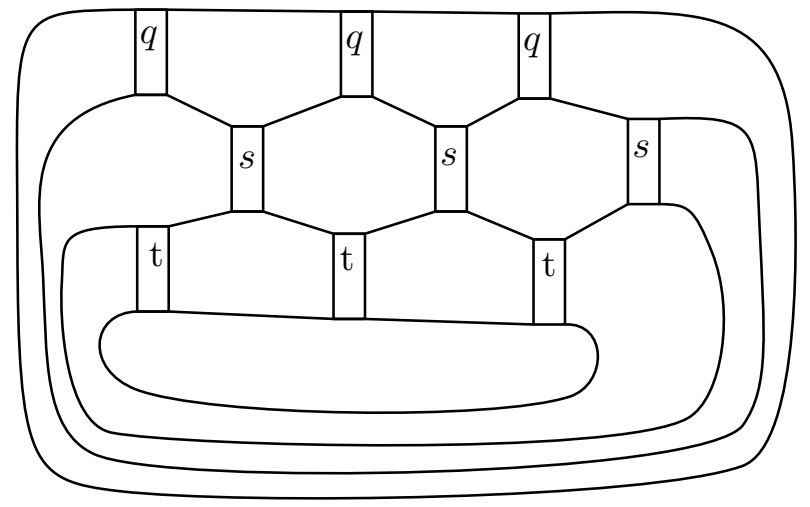

Figure 21. The link $L(l ; 0,0, *)$.

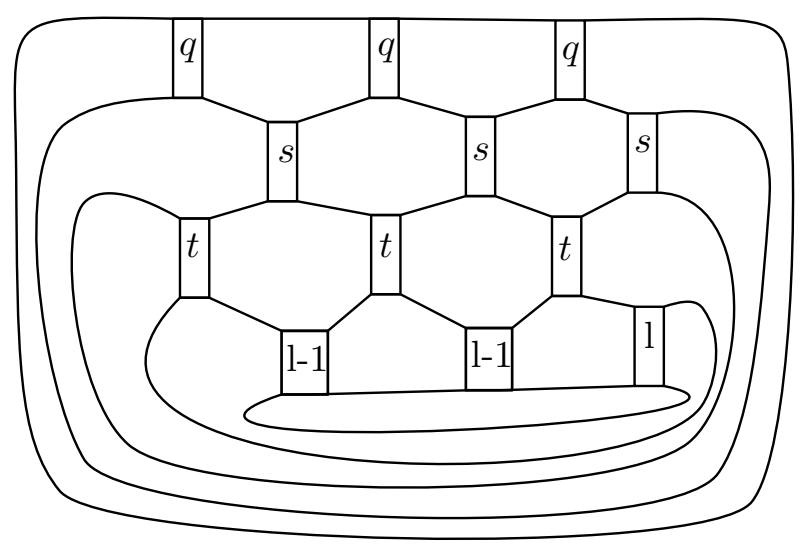

Figure 22 . The link $L(l ; \infty, \infty, *)$. 


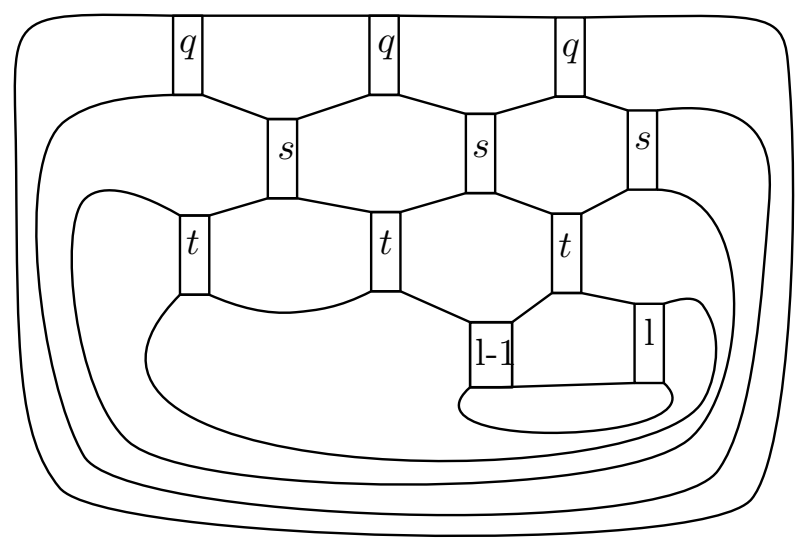

Figure 23. The link $L(l ; 0, \infty, *)$.

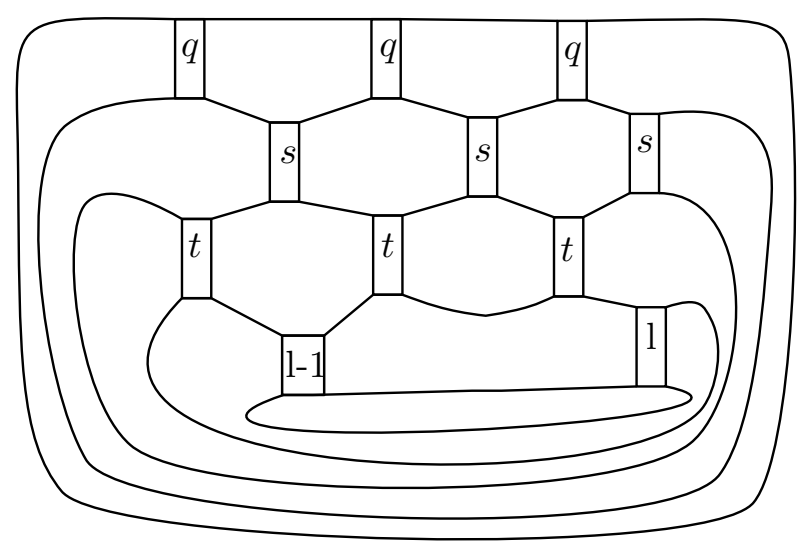

Figure 24. The link $L(l ; \infty, 0, *)$.

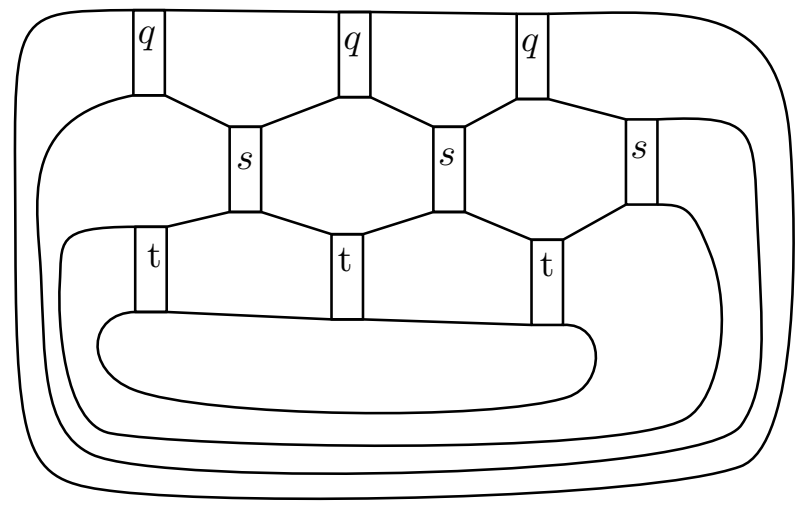

FiguRE 25. The link $L(l ; \infty, 0,0)$. 


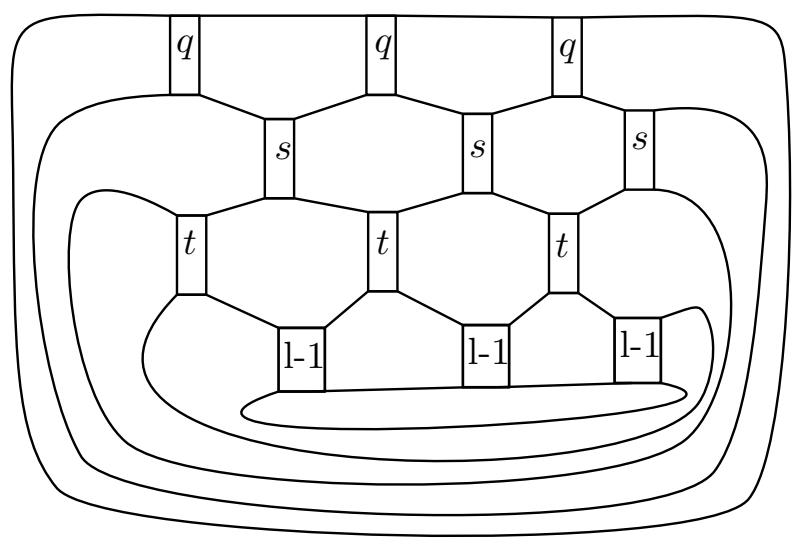

Figure 28. The link $L(l ; \infty, \infty, \infty)$.

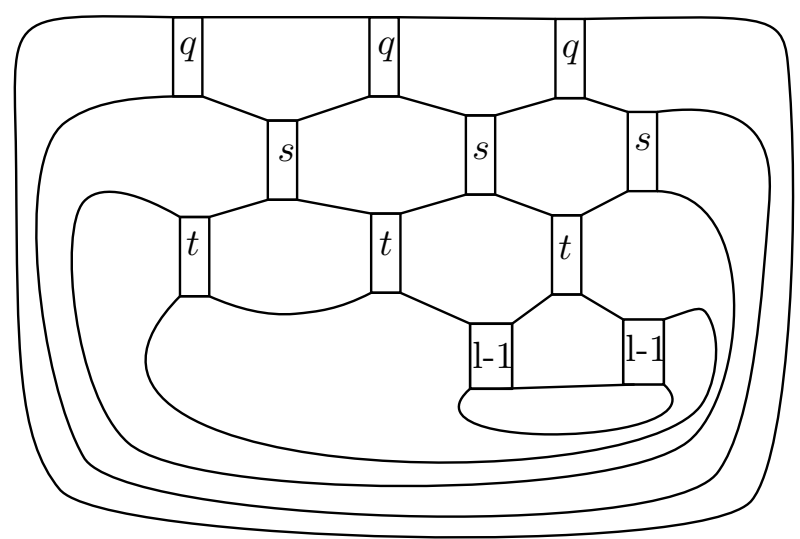

Figure 26. The link $L(l ; 0, \infty, \infty)$.

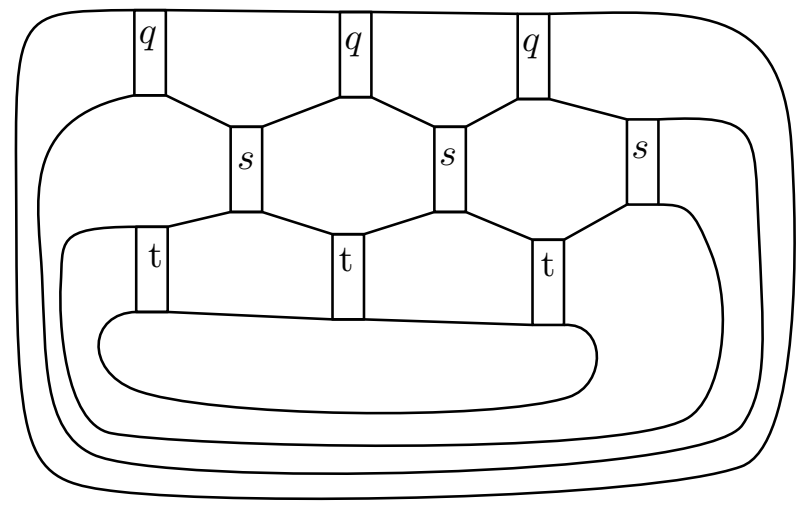

FiguRE 27. The link $L(l ; 0, \infty, 0)$. 


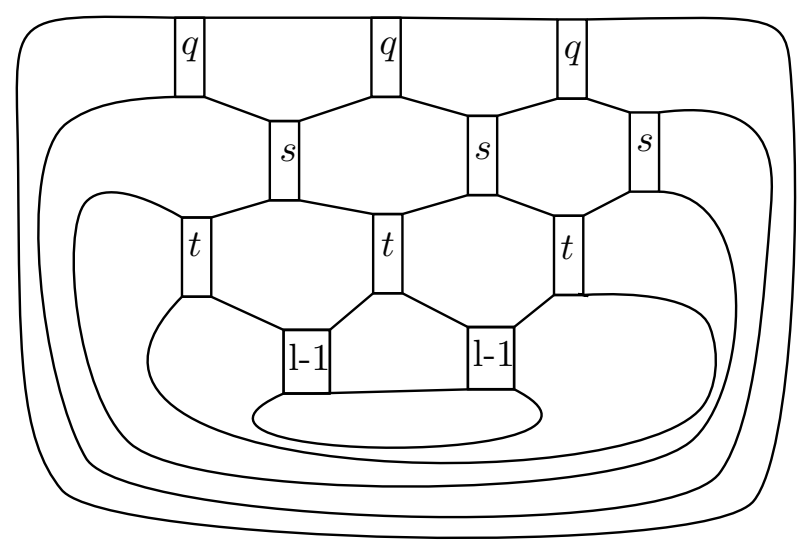

Figure 29. The link $L(l ; \infty, \infty, 0)$.

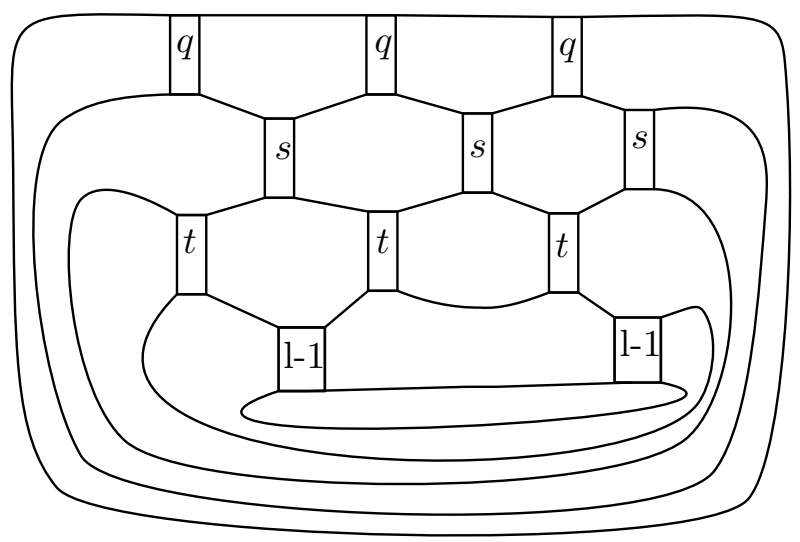

Figure 30 . The link $L(l ; \infty, 0, \infty)$.

The Goeritz matrix of $L$ is

$$
\left[\begin{array}{ccccccccc}
-2 I & I & O & \ldots & O & \ldots & & & \\
I & -2 I & I & O & \ldots & O & \ldots & & \\
\vdots & \vdots & \vdots & \ddots & \vdots & \vdots & \vdots & & \\
O & O \ldots & O & I & P & I & O & \ldots & \\
O & \ldots & O & O & I & -2 I & I & O & \ldots \\
\vdots & \vdots & \vdots & & \vdots & \ddots & \vdots & \vdots & \vdots \\
O & \ldots & O & O & \ldots O & I & -2 I & I & O \\
O & \ldots & O & O & \ldots O & O & I & -2 I & I \\
O & O & O & \ldots & \ldots & O & O & I & Q
\end{array}\right]
$$

where

$$
P=\left[\begin{array}{ccc}
2 s-2 & -s & -s \\
-s & 2 s-2 & -s \\
-s & -s & 2 s-2
\end{array}\right]
$$


and

$$
Q=\left[\begin{array}{ccc}
2 l-1 & -l & -l \\
-l & 2 l-1 & -l \\
-l & -l & 2 l-1
\end{array}\right]
$$

5.2.1. Case $l=1$. Let $B(*, *, *)=L(l=1 ; *, *, *)$.

We have the following table which gives the determinant of the corresponding link.

\begin{tabular}{|l|l|}
\hline Link & Determinant $(t>1)$ \\
\hline$B(*, *, *)$ & $(1-3 q-3 q s-3 t+9 q s t)^{2}$ \\
\hline$B(0, *, *)$ & $2(-q-t+3 q s t)(1-3 q-3 q s-3 t+9 q s t)$ \\
\hline$B(\infty, *, *)$ & $(1-3 q-3 q s-3 t+9 q s t)(1-q-3 q s-t+3 q s t)$ \\
\hline$B(0, \infty, *)$ & $(-q-t+3 q s t)(2-3 q-3 t-6 q s+9 q s t)$ \\
\hline$B(0,0, *)$ & $3(-q-t+3 q s t)^{2}$ \\
\hline
\end{tabular}

TABLE 4

Lemma 5.8. We have the following:

(1) $A(t ; *, *, *)=B(0,0, *), B(\infty, *, *)=A(t ; \infty, \infty, *)$ and $B(0, \infty, *)=A(\infty, *, *)$

(2) If $q=s=t=1$ then $B(*, *, *)=T(3,5)$ and $B(0, *, *)=P(2,-3,-4)$.

Proof. Recall that the link $B(*, *, *)$ is the following:

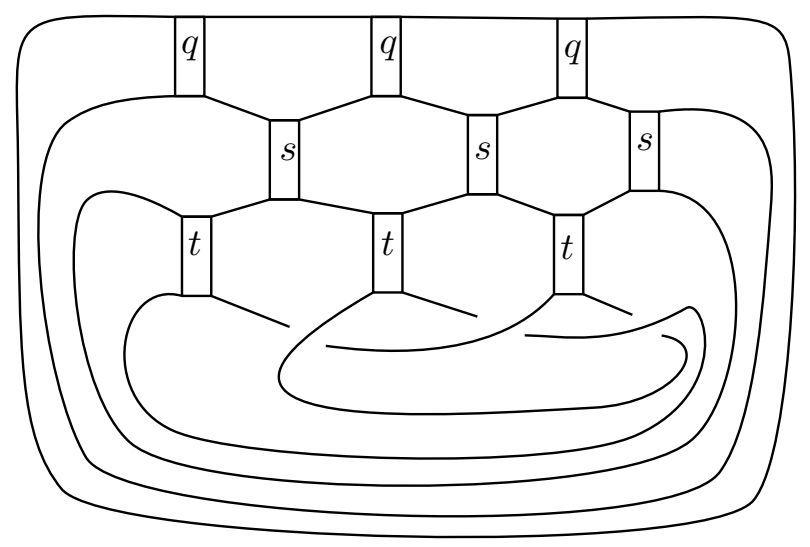

Figure 31 . The link $B(*, *, *)=L(l=1, *, *, *)$.

Therefore, by definition of $B(0,0, *), B(\infty, *, *)$ and $B(0, \infty, *)$, the lemma follows by applying the resolution at the right places.

Lemma 5.9. The Link $B(*, *, *)$ is quasi-alternating for $s>1, t>1$. 
Proof. Since $A(t ; *, *, *)$ and $A(t ; \infty, \infty, *)$ are quasi-alternating for $s>1$ and $t>1$ by Claim 5.5 then $B(0,0, *)$ and $B(\infty, *, *)$ are also quasi-alternating for $s>1$ and $t>1$ by Lemma 5.8. By table 4 , we have that $\operatorname{det} B(*, *, *)=\operatorname{det} B(0, *, *)+\operatorname{det} B(\infty, *, *)$ and $\operatorname{det} B(0, *, *)=$ det $B(0, \infty, *)+\operatorname{det} B(0,0, *)$ for $t>1$, therefore $B(*, *, *)$ is quasi-alternating for $s>1$ and $t>1$.

Lemma 5.10. The 2-fold branched cover of $B(*, *, *)$ and $B(0, *, *)$ are $L$-spaces for $s=1$ and $t>1$.

Proof. First, since $\Sigma_{2}(A(t ; *, *, *))$ and $\Sigma_{2}(A(t ; \infty, \infty, *))$ are L-spaces for $s=1$ by Claim 5.6, then $\Sigma_{2}(B(0,0, *))$ and $\Sigma_{2}(B(\infty, *, *))$ are also L-spaces for $s=1$ by Lemma 5.8. Second, we have that det $B(*, *, *)=\operatorname{det} B(0, *, *)+\operatorname{det} B(\infty, *, *)$ and $\operatorname{det} B(0, *, *)=\operatorname{det} B(0, \infty, *)+$ $\operatorname{det} B(0,0, *)$ for $t>1$ by Table 4 , therefore $\Sigma_{2}(B(0, *, *))$ and $\Sigma_{2}(B(*, *, *))$ are L-spaces for $s=1$ and $t>1$.

5.2.2. Case $l>1$. Proceding similarly as for $A(t ; *, *, *)$, we have the following table which gives the determinant of the corresponding link.

\begin{tabular}{|c|c|}
\hline Link & Determinant $(l>1)$ \\
\hline$L(l ; *, *, *)$ & $(1-3 q l-3 q s-3 l t+9 l q s t)^{2}$ \\
\hline$L(l ; 0, *, *)$ & $2(-t-q+3 q s t)(1-3 l q-3 q s-3 l t+9 l q s t)$ \\
\hline$L(l ; \infty, *, *)$ & $\begin{array}{l}(1-3 l q-3 q s-3 l t+9 l q s t)(1+2 q-3 l q-3 q s+ \\
2 t-3 l t-6 q s t+9 l q s t)\end{array}$ \\
\hline$L(l ; 0, \infty, *)$ & $\begin{array}{l}(-q-t+3 q s t)(2+3 q-6 l q-6 q s+3 t-6 l t- \\
9 q s t+18 l q s t)\end{array}$ \\
\hline$L(l ; \infty, 0, *)$ & $\begin{array}{l}(-q-t+3 q s t)(2+3 q-6 l q-6 q s+3 t-6 l t- \\
9 q s t+18 l q s t)\end{array}$ \\
\hline$L(l ; 0,0, *)$ & $3(-q-t+3 q s t)^{2}$ \\
\hline$L(l ; \infty, \infty, *)$ & $\begin{array}{l}(1+3 q-3 l q-3 q s+3 t-3 l t-9 q s t+9 l q s t)(1+ \\
q-3 l q-3 q s+t-3 l t-3 q s t+9 l q s t)\end{array}$ \\
\hline$L(l ; \infty, \infty, \infty)$ & $(1+3 q-3 l q-3 q s+3 t-3 l t-9 q s t+9 l q s t)^{2}$ \\
\hline$L(l ; 0, \infty, \infty)$ & $\begin{array}{l}2(-q-t+3 q s t)(1+3 q-3 l q-3 q s+3 t-3 l t- \\
9 q s t+9 l q s t)\end{array}$ \\
\hline
\end{tabular}

TABLE 5

Lemma 5.11. (1) $L(l ; 0,0, *)=L(l ; \infty, 0,0)=L(l ; 0, \infty, 0)$,

(2) $L(l ; \infty, 0, \infty)=L(l ; \infty, \infty, 0)=L(l ; 0, \infty, \infty)$,

(3) $L(l ; \infty, \infty, \infty)=L(l-1 ; *, *, *)$,

(4) $L(l ; 0, \infty, \infty)=L(l-1 ; 0, *, *)$,

(5) $L(l ; 0,0, *)=L(l=1 ;, 0,0, *)$. 
Proof. (1) Figure 21, Figure 25 and Figure 27 are the same.

(2) By applying ambient isotopy, one can see that Figure 26, Figure 29 and Figure 30 depict the same links.

(3) Replacing $l$ by $l-1$ in Figure 17 and considering Figure 28 , one can see that $L(l ; \infty, \infty, \infty)$ $=L(l-1 ; *, *, *)$.

(4) Replacing $l$ by $l-1$ in Figure 19 and considering Figure 26, one can see that $L(l ; 0, \infty, \infty)$ $=L(l-1 ; 0, *, *)$.

(5) Figure 21 does not depends on $l$.

Lemma 5.12. (1) $\operatorname{det} L(l ; *, *, *)=\operatorname{det} L(l ; 0, *, *)+\operatorname{det} L(l ; \infty, *, *)$

(2) $\operatorname{det} L(l ; 0, *, *)=\operatorname{det} L(l ; 0, \infty, *)+\operatorname{det} L(l ; 0,0, *)$

(3) $\operatorname{det} L(l ; \infty, *, *)=\operatorname{det} L(l ; \infty, 0, *)+\operatorname{det} L(l ; \infty, \infty, *)$

(4) $\operatorname{det} L(l ; 0, \infty, *)=\operatorname{det} L(l ; 0, \infty, 0)+\operatorname{det} L(l ; 0, \infty, \infty)$

(5) $\operatorname{det} L(l ; \infty, 0, *)=\operatorname{det} L(l ; 0, \infty, 0)+\operatorname{det} L(l ; 0, \infty, \infty)$

(6) $\operatorname{det} L(l ; \infty, \infty, *)=\operatorname{det} L(l ; 0, \infty, \infty)+\operatorname{det} L(l ; \infty, \infty, \infty)$

Proof. See Table 5 .

Proof of Theorem 5.1. The proof of this theorem will be split into 3 claims.

Claim 5.13. If $s>1$ and $t>1$, then the 2-fold branched cover of $L(l ; *, *, *)$ is an $L$-space.

Proof. We have shown that the link $L(l=1 ; *, *, *)=B(*, *, *)$ is quasi-alternating for $s>1$ and $t>1$. By induction on $l$, assume $l>1$ and the link $L(l-1 ; *, *, *)$ is quasi-alternating for $s>1$ and $t>1$. We will show that $L(l ; *, *, *)$ is quasi-alternating for $s>1$ and $t>1$.

Since $L(l ; \infty, \infty, \infty)=L(l-1 ; *, *, *)$ and $L(l ; 0, \infty, \infty)=L(l-1 ; 0, *, *)$ are quasi-alternating by induction hypothesis, then $L(l ; \infty, \infty, *)$ is also quasi-alternating by Lemma 5.12. Since $L(l ; 0,0, *)=L(l=1 ;, 0,0, *)$, and $L(l=1 ;, 0,0, *)$ is quasi-alternating, then $L(l ; 0,0, *)$ is also quasi-alternating. We have that $L(l ; 0,0, *)=L(l ; \infty, 0,0)=L(l ; 0, \infty, 0)$ and $L(l ; \infty, 0, \infty)$ $=L(l ; \infty, \infty, 0)=L(l ; 0, \infty, \infty)$ by Lemma 5.11 , then $L(l ; 0, \infty, *)$ and $L(l ; \infty, 0, *)$ are quasialternating by Lemma 5.12. Therefore, $L(l ; 0, *, *)$ and $L(l ; \infty, *, *)$ are also quasi-alternating by Lemma 5.12 . Finaly the link $L(l ; *, *, *)$ is quasi-alternating by Lemma 5.12 for $s>1$ and $t>1$.

Therefore, the 2 -fold branched cover of $L(l ; *, *, *)$ is an L-space for $s>1$ and $t>1$ by Theorem 2.4 .

Claim 5.14. If $s=t=1$, then the 2-fold branched cover of $L(l ; *, *, *)$ is an $L$-space.

Proof. We have two cases 
(1) Assume first that $l=q$. We have that if $q=s=t=l=1$ then $B(*, *, *)=T(3,5)$ and $B(0, *, *)=P(2,-3,-4)$ by Lemma 5.8 , and $\Sigma_{2}(T(3,5))$ and $\Sigma_{2}(P(2,-3,-4))$ are Lspaces. By induction on $l$, assume $l>1$ and $\Sigma_{2}(L(l-1 ; *, *, *))$ and $\Sigma_{2}(L(l-1 ; 0, *, *))$ are L-spaces. We will show that $\Sigma_{2}(L(l ; 0, *, *))$ and $\Sigma_{2}(L(l ; *, *, *))$ are L-spaces.

Since $L(l ; \infty, \infty, \infty)=L(l-1 ; *, *, *)$ and $L(l ; 0, \infty, \infty)=L(l-1 ; 0, *, *)$ then $\Sigma_{2}(L(l ; \infty, \infty, \infty))$ and $\Sigma_{2}(L(l ; 0, \infty, \infty))$ are L-spaces by induction hypothesis. Therefore $\Sigma_{2}(L(l ; \infty, \infty, *))$ is an L-space by Lemma 5.12 ([OSz]). Since $L(l ; 0,0, *)=L(l=$ $1 ;, 0,0, *)$ and $\Sigma_{2}(L(l=1 ;, 0,0, *))$ is an L-space, then $\Sigma_{2}(L(l ; 0,0, *))$ is also an Lspace. Since $L(l ; 0,0, *)=L(l ; \infty, 0,0)=L(l ; 0, \infty, 0)$ and $L(l ; \infty, 0, \infty)=L(l ; \infty, \infty, 0)$ $=L(l ; 0, \infty, \infty)$ by Lemma 5.11 , then $\Sigma_{2}(L(l ; 0, \infty, *))$ and $\Sigma_{2}(L(l ; \infty, 0, *))$ are L-space by Lemma 5.12 . Therefore, $\Sigma_{2}\left((L(l ; 0, *, *))\right.$ and $\Sigma_{2}(L(l ; \infty, *, *))$ are also L-spaces by Lemma 5.12 . Finaly $\Sigma_{2}(L(l ; *, *, *))$ is an L-space by Lemma 5.12 .

(2) Fix $q$ and assume $l \geq q$. In case (1), we have shown that if $l=q$, then $\Sigma_{2}((L(l ; 0, *, *))$ and $\Sigma_{2}(L(l ; *, *, *))$ are also L-spaces. By induction on $l$, assume $l>q$ and $\Sigma_{2}(L(l-$ $1 ; *, *, *))$ and $\Sigma_{2}(L(l-1 ; 0, *, *))$ are L-spaces. We will show that $\Sigma_{2}(L(l ; 0, *, *))$ and $\Sigma_{2}(L(l ; *, *, *))$ are L-spaces.

Since $L(l ; \infty, \infty, \infty)=L(l-1 ; *, *, *)$ and $L(l ; 0, \infty, \infty)=L(l-1 ; 0, *, *)$ then $\Sigma_{2}(L(l ; \infty, \infty, \infty))$ and $\Sigma_{2}(L(l ; 0, \infty, \infty))$ are L-spaces by induction hypothesis. Therefore $\Sigma_{2}(L(l ; \infty, \infty, *))$ is an L-space by Lemma $5.12([\mathrm{OSz}])$. Since $L(l ; 0,0, *)=L(l=$ $1 ;, 0,0, *)$ and $\Sigma_{2}(L(l=1 ;, 0,0, *))$ is an L-space, then $\Sigma_{2}(L(l ; 0,0, *))$ is also an Lspace. Since $L(l ; 0,0, *)=L(l ; \infty, 0,0)=L(l ; 0, \infty, 0)$ and $L(l ; \infty, 0, \infty)=L(l ; \infty, \infty, 0)$ $=L(l ; 0, \infty, \infty)$ by Lemma 5.11 , then $\Sigma_{2}(L(l ; 0, \infty, *))$ and $\Sigma_{2}(L(l ; \infty, 0, *))$ are L-spaces by Lemma 5.12 . Therefore, $\Sigma_{2}\left((L(l ; 0, *, *))\right.$ and $\Sigma_{2}(L(l ; \infty, *, *))$ are also L-spaces by Lemma 5.12. Finaly $\Sigma_{2}(L(l ; *, *, *))$ is an L-space by Lemma 5.12 for $l \geq q$.

Since $q$ was arbitrary, then this is true for any $l$ and $q$ such that $l \geq q$. Since $l$ and $q$ are symmetric for $L$, then $\Sigma_{2}(L(l ; *, *, *))$ is an L-space for $s=t=1$.

Claim 5.15. If $s=1$ and $t>1$, then $\Sigma_{2}(L(l ; *, *, *))$ is an $L$-space.

Proof. Similarly as the proofs of the two first claims, we will proceed by induction. We have shown that if $s=1$ and $t>1$, then $\Sigma_{2}(L(l=1 ; *, *, *))=\Sigma_{2}(B(*, *, *))$ and $\Sigma_{2}(L(l=$ $1 ; 0, *, *))=\Sigma_{2}(B(0, *, *))$ are L-spaces by Lemma 5.10 . By induction on $l$, assume $l>1$ and $\Sigma_{2}(L(l-1 ; *, *, *))$ and $\Sigma_{2}(L(l-1 ; 0, *, *))$ are L-spaces. We will show that $\Sigma_{2}(L(l ; 0, *, *))$ and $\Sigma_{2}(L(l ; *, *, *))$ are L-spaces.

Since $L(l ; \infty, \infty, \infty)=L(l-1 ; *, *, *)$ and $L(l ; 0, \infty, \infty)=L(l-1 ; 0, *, *)$ then $\Sigma_{2}(L(l ; \infty, \infty, \infty))$ and $\Sigma_{2}(L(l ; 0, \infty, \infty))$ are L-spaces by induction hypothesis. Therefore $\Sigma_{2}(L(l ; \infty, \infty, *))$ is an L-space by Lemma $5.12([\mathrm{OSz}])$. Since $L(l ; 0,0, *)=L(l=1 ;, 0,0, *)$ and $\Sigma_{2}(L(l=1 ;, 0,0, *))$ is an L-space, then $\Sigma_{2}(L(l ; 0,0, *))$ is also an L-space. Since $L(l ; 0,0, *)=L(l ; \infty, 0,0)=$ $L(l ; 0, \infty, 0)$ and $L(l ; \infty, 0, \infty)=L(l ; \infty, \infty, 0)=L(l ; 0, \infty, \infty)$ by Lemma 5.11 , then $\Sigma_{2}(L(l ; 0, \infty, *))$ and $\Sigma_{2}(L(l ; \infty, 0, *))$ are L-space by Lemma 5.12. Therefore, $\Sigma_{2}((L(l ; 0, *, *))$ and $\Sigma_{2}(L(l ; \infty, *, *))$ are also L-spaces by Lemma 5.12 . Finaly $\Sigma_{2}(L(l ; *, *, *))$ is an L-space by Lemma 5.12 . 
Since $s$ and $t$ are symmetric for $L(l ; *, *, *)$, then $\Sigma_{2}(L(l ; *, *, *))$ is also an L-space for $s>1$ and $t=1$.

This complete the proof of Theorem 5.1 by Theorem 5.7 for $q>0, s>0, t>0$ and $l>0$.

(2) If $q<0, s>0, t<0$ and $l>0$ then the link $L$ is alternating, and therefore quasi-alternating, so Theorem 5.1 is a consequence of Theorem 5.7.

(3) If $q>0, s<0, t>0$ and $l>0$ then the link $A$ is alternating and therefore quasi-alternating. Hence the proof of Theorem 5.1 is dealt with as in case (1).

(4) If $q<0, s<0, t<0$ and $l>0$ then the link $A$ is the mirror image of the link $A$ in the case (1), so a similar proof to that used in case (1) can be used to prove this case.

(5) If $q>0, s>0, t<0$ and $l>0$ then as $q$ and $l$ are symmetric for $L(l ; *, *, *)$, and $s$ and $t$ are also symmetric for $L(l ; *, *, *)$, this case of Theorem 5.1 follows as in case $(3)$.

(6) If $q>0, s<0, t<0$ and $l<0$ then as $q$ and $l$ are symmetric for $L(l ; *, *, *)$, and $s$ and $t$ are also symmetric for $L(l ; *, *, *)$, this case of Theorem 5.1 follows as in case (4).

For (7), (8) the proof of Theorem 5.1 is done in a similar way as case (1).

Remark 5.16. (1) In general, it is not true that for every two-bridge knot $K$ the 3 -fold cyclic branched cover is an $L$-space. Boileau-Boyer-Gordon [BBG] have shown that the 3 -fold cyclic branched cover of some familly of strongly quasipositive 2-bridge knots is not an $L$-space. They also show that for some familly of genus 2 strongly quasipositive 2 -bridge knots the 3 -fold cyclic branched cover is an $L$-space.

(2) Claim 5.13 gives an infinite family of quasi-alternating links whose 2 -fold branched cover has none left-orderable fundamental group.

\section{REFERENCES}

[BBG] Michel Boileau, Steven Boyer, Cameron McA. Gordon, Branched covers of quasipositive links and Lspaces, arxiv.

[BGW] Steven Boyer, Cameron McA. Gordon, and Liam Watson On L-spaces and left-orderable fundamental groups, Math. Ann. 356 (2013), 1213-1245.

[BRW] Steven Boyer, Dale Rolfsen, and Bert Wiest, Orderable 3-manifold groups, Ann. Inst. Fourier 55 (2005), $243-288$.

[BZ] Steven Boyer and Xingru Zhang, Cyclic surgery and boundary slopes, Geometric topology, W. Kazez ed., AMS/IP Studies in Advanced Mathematics 2 (1996), 62-79.

[CR] Adam Clay and Dale Rolfsen, Ordered groups and topology, 2015.

[DPT] M. Dabkowski, J. Przytycki, and A. Togha, Non-left-orderable 3-manifold groups, Canadian Math. Bull., 48(1) 32-40, 2005.

[GL] Cameron McA. Gordon, Tye Lidman, Taut foliations, left-orderability, and cyclic branched covers, Acta Math. Vietnam 39 (2014), no.4, 599-635.

[Hu] Ying $\mathrm{Hu}$, The left-orderability and the cyclic branched coverings, Algebraic and Geometric Topology 15 (2015) 399-413.

[K] Taizo Kanenobu, Genus and Kaufman Polynomial of a 2-bridge knot, Osaka J. Math., 29 (1992), 635-651.

[Ka] A. Kawauchi, A survey of knot theory, Birkhauser Verlag, Basel, 1996. 
[L] W.B. Raymond Lickorish, An Introduction to Knot Theory, Graduate texts in mathematics, Springer, 1997.

[MV] Michele Mulazzani and Andrei Vesnin, Generalized Takahashi manifolds, Osaka J. Math. 39 (2002), 705-721.

[OSz] Peter Ozsvath and Zoltan Szabo, On the Heegaard Floer homolgy of branched double-covers, Adv. Math. 194 (2005), 1-33.

[OSz06] Peter Ozsvath and Zoltan Szabo, Introduction to Heegaard Floer theory, Clay Mathematics Institute. 5 (2006), 3-28.

[P] T. Peters, On L-spaces and non left-orderable 3-manifold groups, preprint, arXiv.

[Pr] Jozef H. Przytycki, From Goeritz matrix to quasi-alternating links, preprint, arXiv.

[R] Dale Rolfsen, knots and Links, Publish or Perish, 1976.

[Te] Masakazu Teragaito, Cyclic branched covers of alternating knots and L-spaces, Bull. Korean Math. Soc. 52 (2015), 1139-1148.

[Tra] Anh T. Tran, On left-orderability and cyclic branched coverings, Journal of the Mathematical Society of Japan 67 (2015), no. 3, 1169-1178.

Département de Mathématiques, Université du Québec À Montréal, 201 Avenue du PrésidentKennedy, Montréal, QC H2X 3Y7.

E-mail address: ba.idrissa@courrier.uqam.ca 